\title{
Wideband Extended Range-Doppler Imaging and Waveform Design in the Presence of Clutter and Noise
}

\author{
Birsen Yazıc1, Senior Member, IEEE, and Gang Xie
}

\begin{abstract}
This paper presents a group-theoretic approach to address the wideband extended range-Doppler target imaging and design of clutter rejecting waveforms. An exact imaging method based on the inverse Fourier transform of the affine group is presented. A Wiener filter is designed in the affine group Fourier transform domain to minimize wideband clutter range-Doppler reflectivity. The Wiener filter is then used to form an operator to precondition transmitted waveforms to reject clutter. Alternatively, the imaging and clutter rejection methods are equivalently re-expressed to perform clutter suppression upon reception. These methods are coupled with noise suppression upon reception. Numerical simulations are performed to demonstrate the performance of the proposed approach. Our study shows that the framework introduced in this paper can address the joint design of receive and transmit processing, design of clutter rejecting waveforms, suppression of noise, and reduction of computational complexity in receive processing.
\end{abstract}

Index Terms - Affine group, extended range-Doppler reflectivity, waveform design, wideband, Wiener filter.

\section{INTRODUCTION}

I $\mathrm{N}$ radar and sonar, it is often desirable to image an extended range-Doppler target. This means that the target environment is either composed of several objects or consists of a physically large object corresponding to a continuum of scatterers and that the scatterers are very close in range-Doppler space. Examples of such scenarios include terrain imaging using synthetic aperture radar, underwater imaging using synthetic aperture sonar, and imaging a dense collection of moving targets. An extended range-Doppler target is modeled by a reflectivity density function in the range-Doppler space. The received echo from an extended range-Doppler target is modeled as a weighted sum of delayed and scaled replicas of the transmitted waveform $f$

$$
e(t)=\int_{-\infty}^{\infty} \int_{0}^{\infty} T_{W}(s, \tau) \frac{1}{\sqrt{s}} f\left(\frac{t-\tau}{s}\right) \frac{d s}{s^{2}} d \tau
$$

Manuscript received February 7, 2005; revised May 1, 2006. This work was supported by the Air Force Office of Scientific Research under the agreement FA9550-04-1-0223.

B. Yazıc1 is with the Department of Electrical, Computer and System Engineering, Rensselaer Polytechnic Institute, Troy, NY 12180-3590 USA (e-mail: yazici@ecse.rpi.edu).

G. Xie was with the Department of Mathematical Sciences, Rensselaer Polytechnic Institute, Troy, NY 12180-3590 USA (e-mail: xieg@ rpi.edu).

Communicated by A. Høst-Madsen, Associate Editor for Detection and Estimation.

Color versions of Figs. 5-9 are available online at http://ieeexplore.ieee.org.

Digital Object Identifier 10.1109/TIT.2006.881750 where $\tau$ is the round-trip time delay, $s$ is the Doppler-stretch, and $T_{W}(s, \tau)$ is called the wideband range-Doppler reflectivity density function [4], [9], [20], [21], [28], [36]. The Doppler stretch $s$ is given by $s=(c-v) /(c+v)$, where $c$ is the speed of the transmitted signal and $v$ is the radial velocity of the scatterer. When the transmitted signal is narrowband, the echo model in (1) can be approximated by

$$
e(t)=\int_{-\infty}^{\infty} \int_{-\infty}^{\infty} T_{N}(\omega, \tau) f(t-\tau) e^{i \omega t} d \omega d \tau
$$

where $\omega$ is called the Doppler-shift and $T_{N}(\omega, \tau)$ is called the narrowband range-Doppler reflectivity density function [4], [20], [21]. In general, (1) is referred to as the wideband echo model and (2) is referred to as the narrowband echo model. The narrowband model is sufficient for most radar applications. However, sonar and ultra-wideband radar require the wideband model [6], [29]. In this paper, we will focus on the imaging applications in which the wideband model (1) is required.

Typically in radar and sonar, the echo from a target, such as a moving vehicle, is corrupted with clutter and additive noise. Therefore, the received signal is often processed by matched filtering. When the signal of interest is embedded in white Gaussian noise, matched filtering amounts to matching the received signal to the delayed and scaled replicas of the transmitted signal. This leads to the following wideband imaging equation [5]:

$$
B_{W}(s, \tau)=\int_{-\infty}^{\infty} \int_{0}^{\infty} T_{W}\left(s^{\prime}, \tau^{\prime}\right) A_{W}\left(\frac{s}{s^{\prime}}, \frac{\tau-\tau^{\prime}}{s^{\prime}}\right) \frac{d s^{\prime}}{s^{\prime 2}} d \tau^{\prime}
$$

where $B_{W}$ is the wideband cross-ambiguity function of the transmitted waveform and the received echo and $A_{W}$ is the wideband auto-ambiguity function of the transmitted waveform. Note that the wideband cross-ambiguity function in (3) is the convolution of the wideband target reflectivity density function and the auto-ambiguity function over the affine group.

The imaging equation (3) shows that the resolution of the matched filter output is limited by the auto-ambiguity function of the transmitted waveform, which plays the role of a point-spread function for the range-Doppler imaging system. Since the auto-ambiguity function is formed by the transmitted waveform, one can attempt to design a waveform to improve the resolution of reconstructed images. However, fairly strong constraints on the mathematical form of the auto-ambiguity function precludes synthesis of an ideal auto-ambiguity function, i.e., a Dirac-delta function located at the origin of the rangeDoppler space. 
In this paper, we present a mathematical framework based on the Fourier theory of the affine group to address the joint design of image reconstruction and waveform design for clutter rejection to recover the wideband range-Doppler target reflectivity density in the presence of noise and waveform-dependent clutter. We observe that the forward map in (1) is a bilinear operator that acts on both the transmitted waveform and the target reflectivity density function. In particular, this operator maps the target reflectivity density function to an operator by the Fourier transform of the affine group, which in turn acts on the transmitted waveform to produce the echo signal. Therefore, our imaging equation is based on inverting the affine group Fourier transform of the wideband reflectivity density function. Unlike the standard Fourier transform, the affine group Fourier transform of a function is operator valued, i.e., each Fourier component is an operator. Therefore, transmitting a single waveform allows only one of the matrix coefficients of the spectral operator to be measured in received echo. However, affine group Fourier inversion shows that all the matrix coefficients of the spectral operator over which the target lies have to be measured in received echo. Therefore, our approach shows that if multiple pulses are transmitted, the target can be reconstructed exactly, leading to Dirac-delta point-spread function for the imaging equation in range-Doppler domain. When the echo from the target is embedded in unwanted clutter echo, we design a Wiener operator in the affine group Fourier transform domain based on the minimum mean-square error criteria to minimize the clutter. We show that if the target and clutter random fields exhibit stationarity under the affine group translations, the Wiener operator becomes a convolution filter over the affine group. Using the Wiener filter and the discrepancy operator of the affine group Fourier transform, we form another operator, which we refer to as the preconditioning operator, to condition the transmitted waveforms to reject clutter. We present two algorithms for imaging and clutter rejection/suppression: one for clutter rejection in transmit by means of the preconditioning operator, and one for clutter suppression upon reception by implementing the preconditioning operator in receive. These two algorithms are equivalent in the mean-square error sense; however, clutter rejection in transmit is computationally more efficient than the clutter suppression in receive. We also provide a third algorithm that combines clutter rejection in transmit and additive noise suppression in receive. The image reconstruction method introduced in this paper can be also viewed as a data fusion method to synthesize high-resolution range-Doppler images from multiple coherent narrowband radars.

The rest of the paper is organized as follows: Section II provides a review of the related work. Section III introduces the Fourier theory of the affine group. Section IV provides the problem formulation. Section V presents the derivation of the preconditioning operator for the design of clutter rejecting waveforms. Section VI presents the estimation of the range-Doppler reflectivity in the presence of noise and clutter and the design of clutter rejecting waveforms. Section VII presents numerical experiments to validate our approach. Section VIII summarizes our results and draws conclusions based on these results. The paper concludes with four appendices.
The first provides a review of the basic concepts from the group representation theory and a review of the Fourier transforms over groups. The second provides a lemma which is used repeatedly in the paper. The third and fourth appendices provide derivations of the results stated in Section VI.

\section{RELATED WORK}

The wideband echo model has been studied by many researchers [19], [21], [24], [36]. In [21], affine group Fourier theory was utilized and a method of waveform design and wideband range-Doppler target reflectivity reconstruction was introduced. A similar approach was also noted in [19]. In [24], the approach in [21] was extended to include frames as a set of transmitted waveforms. In [36], the use the wavelet transform for the image recovery problem was proposed in a deterministic setting. However, this approach requires the target reflectivity function to be in the reproducing kernel Hilbert space of the transmitted mother wavelet. In all of these studies, [19], [21], [24], and [36], the received echo is modeled in a deterministic setting without any consideration of noise and clutter in sensor measurements. Our study is closest in spirit to [21], however, our problem is to design clutter rejecting waveforms and to estimate the wideband range-Doppler target reflectivity density function embedded in clutter using a priori statistical information.

In the area of waveform design, there is a large body of literature based on the classical concept of ambiguity and matched filtering for the narrowband echo model [2], [7], [8], [11], [16], [17], [25], [26], [37]. These studies address the design of sequence of waveforms having an ambiguity function with narrow central main lobe surrounded by uniform low-level sidelobes to approximate the ideal Dirac-delta like ambiguity function in range-Doppler space. However, a number of researchers argued that it may not be possible to achieve the desired resolution or discrimination capability using a single-pulse echo system based on classical matched filtering [18], [25], [26]. In [18] and [25], the concept of composite or combined ambiguity function derived from the coherent sum of multiple classical matched filtering was introduced and diversity phase-, frequency-, and joint phase-frequency-coded waveforms were presented for narrowband echo model.

In [3], [12]-[15], [23], and [27], the classical concept of matched filtering was further developed to address the joint design of optimum waveforms and receive processing for target detection and classification applications. In [3], [12], [13], and [23], methods of waveform/receiver design have been developed for target detection based on maximizing the signal-to-noise or signal-to-interference ratios. These studies assume that the target is range-only extended target and the impulse response function of the target is known and deterministic. In [12], [23] was extended to target identification problem. In [3], design of optimal waveforms for target classification based on mutual information criteria for range-only extended statistical target model was studied. In [27], a discretized version of the wideband target reflectivity model was used, and a method of waveform design for target detection and classification was introduced based on the Kullback-Leibler measure. Note that while the studies cited above are close in spirit to our work in 
that they both address the joint design of transmit and receive processing, our problem is focused primarily on the design of imaging waveforms rather than detection or classification waveforms. Therefore, we use the mean-square error criterion as a figure of merit for optimality instead of the measures designed for the output of a discriminant functional used in a detector or classifier. Additionally, the aforementioned studies assume that the target reflectivity density function is known and deterministic. However, in our problem the range-Doppler target reflectivity density function is unknown and our objective is to estimate this function embedded in clutter by designing clutter rejecting transmit waveforms and receive processing with respect to the mean-square error criteria. Therefore, we use statistical a priori information on the unknown wideband range-Doppler target and clutter reflectivity density functions.

\section{Fourier THEORY OF THE AfFINE GROUP}

In Appendix I, we provide a brief overview of the Fourier analysis over groups and definitions of basic concepts. In this section, we will introduce the affine group and summarize the Fourier analysis over the affine group from a perspective relevant to the rest of our discussion. A detailed discussion on the Fourier theory of the affine group can be found in [30].

Below we provide an index of notation used throughout the paper. We use upper case letters, $F, T$, etc., for functions defined on the affine group and lower case letter $f, \varphi$, etc., for functions defined on $\mathbb{R}$. The operators are denoted by upper case Greek or caligraphic letters, e.g., $\mathcal{A}, \mathcal{F}, \mathcal{S}, \Pi, \Xi$.

\section{NOMENCLATURE}

$g=(s, \tau)$

$g_{1} g_{2}$

$\mathbb{A}=\mathbb{R} \ltimes \mathbb{R}^{+}$

$L^{2}(\mathrm{~A}, d g)$

$L^{1}(\mathrm{~A}, d g)$

$d g=d_{L} g$ $=s^{-2} d s d \tau$

$d_{R}(g)=s^{-1} d s d \tau$

$\Pi_{ \pm}$

$H_{ \pm}$

$\left\{\varphi_{ \pm}^{n}\right\}$

$\mathcal{F}_{ \pm}(F)$

$\Pi_{ \pm}^{\dagger}$
Elements of the affine group.

Affine group operation .

Affine group .

Space of square summable functions on $A$.

Space of absolutely summable functions on $A$.

Left-Haar measure of $A$.

Right-Haar measure of $A$.

Unitary irreducible group representations of $A$.

Space of $L^{2}(\mathbb{R}, d t)$ functions whose Fourier transform is supported on $\mathbb{R}^{ \pm}$.

Differentiable orthonormal basis on $H_{ \pm}$.

Affine group Fourier components of F.

Adjoint of $\Pi_{ \pm}$.
$\Xi_{ \pm}$

$*$ A

Discrepancy operators of the affine group .

Convolution over the affine group.

\section{A. Affine Group}

The affine group, or the $s x+\tau$ group, is a two-parameter Lie group whose elements are given by $2 \times 2$ matrices of the form

$$
\left[\begin{array}{ll}
s & \tau \\
0 & 1
\end{array}\right], \quad s \in \mathbb{R}^{+}, \tau \in \mathbb{R}
$$

parameterized by the scale parameter $s$ and the translation parameter $\tau$.

Let $g$ denote $(s, \tau)$, the elements of the affine group. The affine group operation is given by the usual matrix multiplication, i.e., $g_{1} g_{2}=\left(s_{1}, \tau_{1}\right)\left(s_{2}, \tau_{2}\right)=\left(s_{1} s_{2}, s_{1} \tau_{2}+\tau_{1}\right)$; and the inverse elements are given by the matrix inversion $(s, \tau)^{-1}=\left(s^{-1},-s^{-1} \tau\right)$. This defines the affine group as a semidirect product of the additive group $(\mathbb{R},+)$ and the multiplicative group $\left(\mathbb{R}^{+}, \times\right)$, i.e., $\mathbb{R} \ltimes \mathbb{R}^{+}$. For the rest of the paper, we shall denote the affine group by $\mathbb{A}$. Note that the affine group is noncommutative.

Let $L^{2}(\mathcal{A}, d g)$ and $L^{1}(\mathcal{A}, d g)$ denote the space of square summable and absolutely summable functions over $\mathbb{A}$, respectively, i.e.,

$$
\int_{\text {A }}|F(g)|^{2} d_{L} g<+\infty, \quad \int_{\text {A }}|F(g)| d_{L} g<+\infty
$$

where $d_{L} g=s^{-2} d s d \tau$ is the left-Haar measure of the affine group. The inner product of two functions $F_{1}$ and $F_{2}$ in $L^{2}(\mathrm{~A}, d g)$ is defined as

$$
\left\langle F_{1}, F_{2}\right\rangle=\int_{\text {A }} F_{1}(g) \overline{F_{2}(g)} d g .
$$

Note that the affine group is a nonunimodular group. Therefore, the right-Haar measure is given by $d_{R} g=s^{-1} d s d \tau$. The right- and left-Haar measures of a locally compact group are related by $d_{L}(g)=\Delta(g) d_{R}(g)$, where $\Delta(g)$ is the modular function of the group. For the affine group $\Delta(g)=s^{-1}$. In the rest of the paper, we shall use the left-Haar measure and denote it by $d g$ to simplify our notation.

\section{B. Fourier Transform Over the Affine Group}

The standard Fourier transform, which is defined with respect to the additive group $(\mathbb{R},+)$, projects functions in $L^{2}(\mathbb{R}, d t)$ onto complex exponentials $e^{i \omega}, \omega \in \mathbb{R}$. In the general theory of Fourier analysis over groups, square summable functions defined on the group are projected onto the unitary irreducible representations of the group. For commutative groups, unitary irreducible representations are one-dimensional scalar-valued operators; however, for noncommutative groups, they are either finite-dimensional matrices or infinite-dimensional operators. As a result, the Fourier components of a function defined on a noncommutative group are either matrix or operator valued. In the case of the affine group, there are two nonequivalent, infinite-dimensional, irreducible, unitary representations. Hence, there are two Fourier components; each, however, can be viewed as an infinite-dimensional matrix. As we will show, each transmitted 
waveform provides information about one of the matrix elements of the Fourier components.

\section{Irreducible Unitary Representations of the Affine Group}

Let $\Pi_{+}$and $\Pi_{-},(s, \tau) \in A$ denote the two irreducible, unitary representations of the affine group. In one realization, for each element $(s, \tau)$ of the affine group, the linear operator $\Pi_{+}(s, \tau)$ acts on the Hilbert space $H_{+}$, which consists of functions $\varphi_{+}$whose Fourier transforms are supported on the positive real line. Similarly, $\Pi_{-}$acts on $H_{-}$, the orthogonal complement of $H_{+}$, which consists of functions $\varphi_{-}$whose Fourier transforms are supported on the negative real line.

Note that $L^{2}(\mathbb{R}, d t)$ is a direct sum of $H_{+}$and $H_{-}$, i.e., $L^{2}(\mathbb{R})=H_{+} \oplus H_{-}$. The linear operators

$$
\Pi_{ \pm}(s, \tau) \varphi_{ \pm}(t)=\frac{1}{\sqrt{s}} \varphi_{ \pm}\left(\frac{t-\tau}{s}\right)
$$

are unitary, nonequivalent, and irreducible in the spaces $H_{+}$and $H_{-}$, respectively.

\section{The Fourier Transform of the Affine Group}

The affine Fourier transform of a function $f \in L^{2}(A, d g)$ is defined as

$$
\mathcal{F}_{ \pm}(F)=\int_{-\infty}^{\infty} \int_{0}^{\infty} s^{-2} d s d \tau F(s, \tau) \Pi_{ \pm}(s, \tau),(s, \tau) \in \text { A. }
$$

Note that the Fourier components $\mathcal{F}_{ \pm}(F)$ are operators. For a given orthonormal basis $\left\{\varphi_{ \pm}^{n}(t)\right\}$ of $H_{ \pm}$, the $n m$ th matrix coefficients of $\mathcal{F}_{ \pm}(F)$ is given by

$$
\begin{aligned}
& \mathcal{F}_{ \pm}(F)_{n m}=\left\langle\mathcal{F}_{ \pm}(F) \varphi_{ \pm}^{n}, \varphi_{ \pm}^{m}\right\rangle \\
& =\int_{-\infty}^{\infty} \int_{-\infty}^{\infty} \int_{0}^{\infty} F(s, \tau) \frac{1}{\sqrt{s}} \varphi_{ \pm}^{n}\left(\frac{t-\tau}{s}\right) \overline{\varphi_{ \pm}^{m}(t)} s^{-2} d s d \tau d t .
\end{aligned}
$$

\section{E. The Inverse Fourier Transform of the Affine Group}

The inverse affine Fourier transform is given by

$$
F(s, \tau)=\sum_{ \pm} \operatorname{trace}\left(\Pi_{ \pm}^{\dagger}(s, \tau) \mathcal{F}_{ \pm}(F) \Xi_{ \pm}\right), \quad(s, \tau) \in \text { A }
$$

where $\Pi_{ \pm}^{\dagger}(s, \tau)$ denotes the adjoint of $\Pi_{ \pm}(s, \tau)$; and $\Xi_{ \pm}$are linear, self-adjoint, positive-definite operators defined as

$$
\Xi_{ \pm} \varphi_{ \pm}(t)=\mp \frac{i}{2 \pi} \frac{d \varphi_{ \pm}}{d t}(t) .
$$

Note that the inverse Fourier transform with respect to the affine group is a filtered backprojection operator. It requires the Fourier components $\mathcal{F}_{ \pm}(F)$ to be filtered by the operators $\Xi_{ \pm}$first; and then maps the result back to $L^{2}($ A,$d g)$ by the adjoint $\Pi_{ \pm}^{\dagger}(s, \tau)$ of the forward Fourier transform to synthesize the function $F$. The filtering in the inverse Fourier transform of the affine group arises due to the non-unimodular nature of the affine group. The standard inverse Fourier transform, on the other hand, is given by a backprojection operator only due to the commutative (hence, unimodular) nature of the additive group. We will use the operators $\Xi_{ \pm}$repeatedly for the rest of the paper. For ease of referencing, we shall refer to them as the discrepancy operators of the affine Fourier transform. The term discrepancy alludes to the difference between the forward Fourier transform and its adjoint, i.e, the backprojection operator.

For a given set of $\left\{\varphi_{ \pm}^{n}(t)\right\}$ orthonormal, differentiable basis for $H_{ \pm}$, the inverse affine Fourier transform can be alternatively expressed as

$$
F(s, \tau)=\sum_{ \pm} \sum_{n}\left\langle\Pi_{ \pm}^{\dagger}(s, \tau) \mathcal{F}_{ \pm}(F) \Xi_{ \pm} \varphi_{ \pm}^{n}, \varphi_{ \pm}^{n}\right\rangle .
$$

\section{F. Sum of the Affine Fourier Components}

For ease of notation, we define the following operators:

$$
\begin{aligned}
\Pi(s, \tau) & =\Pi_{+}(s, \tau) \oplus \Pi_{-}(s, \tau), \quad(s, \tau) \in \text { A } \\
\Xi & =\Xi_{+} \oplus \Xi_{-} .
\end{aligned}
$$

Then, we can express the sum of the two Fourier components given in (8) as follows:

$$
\begin{aligned}
\mathcal{F}(F) & =\mathcal{F}_{+}(F) \oplus \mathcal{F}_{-}(F) \\
& =\int_{-\infty}^{\infty} \int_{0}^{\infty} F(s, \tau) \Pi(s, \tau) s^{-2} d s d \tau,(s, \tau) \in \text { A. }
\end{aligned}
$$

The inverse Fourier transform given in (10) and (12) can be reexpressed as

$$
\begin{aligned}
F(s, \tau) & =\operatorname{trace}\left(\Pi^{\dagger}(s, \tau) \mathcal{F}(F) \Xi\right), \quad(s, \tau) \in \text { A } \\
& =\sum_{n}\left\langle\mathcal{F}(F) \Xi \varphi^{n}, \Pi(s, \tau) \varphi^{n}\right\rangle
\end{aligned}
$$

where $\varphi^{n}(t)=\varphi_{+}^{n}(t)+\varphi_{-}^{n}(t)$, and $\left\{\varphi_{ \pm}^{n}(t)\right\}$ are orthonormal, differentiable basis for $H_{ \pm}$.

\section{G. Convolution Over the Affine Group}

The convolution of two functions $F_{1}, F_{2}$ over the affine group is given by

$$
\begin{array}{r}
\left(F_{1} *_{\mathrm{A}} F_{2}\right)(s, \tau)=\int_{-\infty}^{\infty} \int_{0}^{\infty} F_{1}\left(s^{\prime}, \tau^{\prime}\right) F_{2}\left(\frac{s}{s^{\prime}}, \frac{\tau-\tau^{\prime}}{s^{\prime}}\right) \\
s^{\prime-2} d s^{\prime} d \tau^{\prime}, \quad(s, \tau) \in \text { A. }
\end{array}
$$

Analogous to the standard Fourier transform, under the affine group Fourier transform, the convolution of two functions over the affine group becomes operator multiplication. More specifically

$$
\mathcal{F}_{ \pm}\left(F_{1} *_{\AA} F_{2}\right)=\mathcal{F}_{ \pm}\left(F_{1}\right) \mathcal{F}_{ \pm}\left(F_{2}\right) .
$$

In the following sections, we will use the convolution property (17) of the affine group to design appropriate filters in the Fourier domain to suppress clutter and noise. 


\section{PROBLEM FORMULATION}

\section{A. Deterministic Formulation}

We model the echo signal from the target as the sum of the affine Fourier components of the target reflectivity density function evaluated at the transmitted waveform, i.e.,

$$
e_{T}(t)=\mathcal{F}(T) f(t)
$$

where $f$ is the transmitted pulse and $T$ is the wideband target reflectivity density function.

The ideal imaging problem involves recovering the target reflectivity density function $T$ given the transmitted waveform $f$ and the received echo $e_{T}$. Following the conventional rangeDoppler image formation approach, the received echo $e_{T}$ can be affine matched filtered with the transmitted waveform $f$ to obtain an estimate of $T$, i.e.,

$$
\hat{T}(s, \tau)=\int_{-\infty}^{\infty} e_{T}(t) \frac{1}{\sqrt{s}} \overline{f\left(\frac{t-\tau}{s}\right)} d t .
$$

Using the notation introduced in Section III, (19) can be expressed as

$$
\hat{T}(s, \tau)=\langle\mathcal{F}(T) f, \Pi(s, \tau) f\rangle
$$

Note that (20) is equivalent to backprojection $e_{T}$ by $\Pi(s, \tau)$. Instead of the conventional image formation approach in (20), we can use the Fourier theory of the affine group and recover a band-limited version of the target reflectivity density function $\hat{T}_{b}$ as follows:

$$
\hat{T}_{b}(s, \tau)=\left\langle\mathcal{F}(T) f, \Pi(s, \tau) \Xi^{-1} f\right\rangle
$$

where $\Xi^{-1}$ denotes the inverse of the operator $\Xi$. (Note that $\Xi^{-1}$ exists since $\Xi$ is a positive definite operator.) Equation (21) shows that the affine matched filtering has to be performed not with the transmitted waveform $f$ but a filtered version of $f$, namely, with $\Xi^{-1} f$. Alternatively, we can choose a baseline signal $\varphi$ and precondition it with the operator $\Xi$ to form the transmitted waveform $f=\Xi \varphi$ and perform the affine matched filtering with $\varphi$ at receive to recover the band-limited version of the target image, i.e.,

$$
\hat{T}_{b}(s, \tau)=\langle\mathcal{F}(T) \Xi \varphi, \Pi(s, \tau) \varphi\rangle .
$$

Note that in (21) the transmitted waveform is filtered at receive and the resulting waveform is then used to perform affine matched filtering with the received echo. In (22), on the other hand, a baseline signal is chosen and filtered at transmit to form the transmitted waveform and the affine matched filtering is performed with the baseline signal at receive. In both cases, unlike the conventional image formation approach, the received echo is matched not to the transmitted waveform, but to a filtered version of it. Clearly, in both schemes the transmitted waveform has to be chosen in the orthogonal complement of the null space of the operator $\mathcal{F}(T)$, i.e., the transmitted waveform has to illuminate the spectral modes of $T$.
For an exact reconstruction of the target reflectivity density function $T$, the Fourier theory of the affine group shows that multiple waveforms need to be transmitted. Extending on (22), we could choose an orthonormal, differentiable basis $\left\{\varphi_{ \pm}^{n}(t)\right\}$ for $H_{ \pm}$and form the transmitted waveforms $f^{n}$ from the filtered versions of $\varphi^{n}$ by $f^{n}=\Xi \varphi^{n}$, where $\varphi^{n}(t)=\varphi_{+}^{n}(t)+\varphi_{-}^{n}(t)$. Then, the target image can be formed by using (15)

$$
T(s, \tau)=\sum_{n}\left\langle\mathcal{F}(T) f^{n}, \Pi(s, \tau) \varphi^{n}\right\rangle
$$

which amounts to the affine matched filtering at the receiver with the baseline signals $\varphi^{n}$ and coherently summing over all the affine matched filter outputs. Clearly, transmitting multiple waveforms facilitates illumination of all the matrix elements of the spectral operator $\mathcal{F}(T)$ over which the target $T$ lies.

Unlike the conventional imaging approach, when (23) is used for image formation, the point spread function of the imaging equation is a Dirac-delta function located at the origin of the affine group. This is due to the additional filtering required by the operator $\Xi$ for the inverse Fourier transform of the affine group. As shown in (21) and (22), the filtering can be performed either in transmit or in receive. In the statistical formulation of the problem, this filter will be replaced with the one designed to suppress clutter based on the second-order statistics of clutter and target fields.

\section{B. Statistical Formulation}

We model all unwanted, spurious background reflections as clutter/interference. It is well known that the echo from the clutter/interference is waveform dependent [23]. Thus, we model the clutter echo as the affine Fourier transform of the clutter reflectivity density function evaluated at the transmitted waveform, i.e.,

$$
e_{C}(t)=\mathcal{F}(C) f(t)
$$

where $C$ is the clutter reflectivity density function. We also assume that the measurements are contaminated with zero mean additive white noise process. As a result, the received signal model is

$$
y(t)=[\mathcal{F}(T+C) f](t)+\xi(t)
$$

where $\xi(t)$ is the zero mean additive white noise with autocorrelation function $\mathbb{E}\left[\xi\left(t_{1}\right) \overline{\xi\left(t_{2}\right)}\right]=\sigma^{2} \delta\left(t_{1}-t_{2}\right)$.

We assume that the target and clutter reflectivity fields are uncorrelated, i.e.,

$$
\mathbb{E}\left[T\left(s_{1}, \tau_{1}\right) \overline{C\left(s_{2}, \tau_{2}\right)}\right]=0, \quad \text { for all }\left(s_{1}, \tau_{1}\right),\left(s_{2}, \tau_{2}\right) \in \text { A. }
$$

Additionally, we assume that the target and clutter echo are uncorrelated with the additive white noise, i.e.,

$$
\begin{aligned}
& \mathbb{E}\left[e_{T}(t) \overline{\xi(t)}\right]=0 \\
& \mathbb{E}\left[e_{C}(t) \overline{\xi(t)}\right]=0, \quad t \in \mathbb{R} .
\end{aligned}
$$

Fig. 1 displays the components of the range-Doppler echo model. 


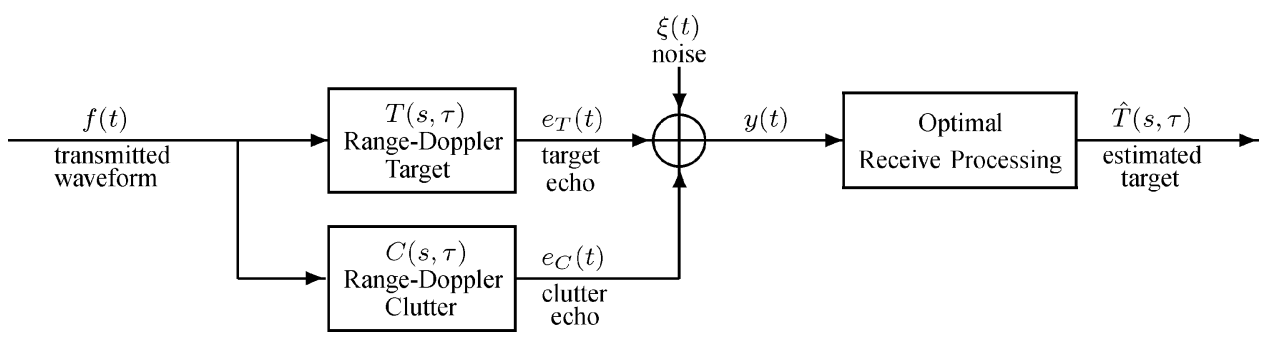

Fig. 1. A block diagram of the range-Doppler echo model.

For simplicity, let us assume that the received echo is due to target and clutter random fields only, i.e.,

$$
e(t)=\mathcal{F}(T+C) f(t) .
$$

If we perform the inverse Fourier transform over the affine group, we obtain

$$
\begin{aligned}
T(s, \tau)+C(s, \tau) & =\operatorname{trace}\left(\Pi^{\dagger}(s, \tau) \mathcal{F}(T+C) \Xi\right), \\
& =\sum_{n}\left\langle\mathcal{F}(T+C) \Xi \varphi^{n}, \Pi(s, \tau) \varphi^{n}\right\rangle
\end{aligned}
$$

where $\left\{\varphi_{ \pm}^{n}(t)\right\}$ are orthonormal, differentiable basis for $H_{ \pm}$and $\varphi^{n}(t)=\varphi_{+}^{n}(t)+\varphi_{-}^{n}(t)$. Ideally, we want to design a filter/ operator $\mathcal{W}$ in the Fourier domain of the affine group so that

$$
\mathcal{F}(\hat{T})=\mathcal{F}(T+C) \mathcal{W} .
$$

Equivalently, in range-Doppler scale domain

$$
\begin{aligned}
\hat{T}(s, \tau) & =\operatorname{trace}\left(\Pi^{\dagger}(s, \tau) \mathcal{F}(T+C) \mathcal{W} \Xi\right), \\
& =\sum_{n}\left\langle\mathcal{F}(T+C) \mathcal{W} \Xi \varphi^{n}, \Pi(s, \tau) \varphi^{n}\right\rangle .
\end{aligned}
$$

Therefore, the idea is to precondition the set of baseline signals $\left\{\varphi^{n}\right\}$ by the filter $\mathcal{W} \Xi$ to design clutter rejecting waveforms $\left\{f^{n}\right\}$ where

$$
f^{n}=\mathcal{W} \Xi \varphi^{n} .
$$

We shall call the operator $\mathcal{W} \Xi$, the preconditioning operator. Note that the operator $\mathcal{W}$ of the preconditioning operator is for clutter rejection and the operator $\Xi$ is for the exact reconstruction. Therefore, we shall use the term preconditioning operator interchangeably for both $\mathcal{W} \Xi$ and $\mathcal{W}$. In the following section, we will discuss the design of such an operator based on the minimum mean-square error criteria.

\section{DESIGN OF PRECONDITIONING OPERATOR FOR ClutTer ReJeCtion}

In this section, we shall first develop a preconditioning operator for nonstationary target and clutter fields based on the minimum mean-square error criteria. Next, we will show that when target and clutter fields exhibit stationarity with respect to the left translations of the affine group, the preconditioning operator becomes a convolution filter over the affine group. Furthermore, the Fourier transform of the preconditioning operator can be expressed in terms of the spectral density operators of target and clutter fields.

\section{A. Nonstationary Target and Clutter Fields}

Let $\mathcal{L}\left(L^{2}(\mathbb{R}, d t)\right)$ denote the Hilbert space of Hilbert-Schmidt operators from $L^{2}(\mathbb{R}, d t)$ to $L^{2}(\mathbb{R}, d t)$, equipped with the norm

$$
\langle\mathcal{A}, \mathcal{B}\rangle=\operatorname{trace}\left(\mathcal{B}^{\dagger} \mathcal{A}\right), \quad \mathcal{A}, \mathcal{B} \in \mathcal{L}\left(L^{2}(\mathbb{R}, d t)\right)
$$

where $\|\mathcal{A}\|^{2}<+\infty$ and $\|\mathcal{B}\|^{2}<+\infty$.

Let $\mathcal{L}_{\mathcal{R}}\left(L^{2}(\mathbb{R}, d t)\right)$ denote the Hilbert space of bounded random operators from $L^{2}(\mathbb{R}, d t)$ to $L^{2}(\mathbb{R}, d t)$, i.e., $\mathcal{C}, \mathcal{D} \in \mathcal{L}_{\mathcal{R}}\left(L^{2}(\mathbb{R}, d t)\right), \mathcal{C}, \mathcal{D}: L^{2}(\mathbb{R}, d t) \rightarrow L^{2}(\mathbb{R}, d t)$, and

$$
\langle\mathcal{C}, \mathcal{D}\rangle_{R}=\operatorname{trace}\left(\mathbb{E}\left[\mathcal{C}^{\dagger} \mathcal{D}\right]\right),
$$

$\|\mathcal{C}\|^{2}<+\infty$ and $\|\mathcal{D}\|^{2}<+\infty$.

Theorem 5.1: Let $T(s, \tau)$ and $C(s, \tau),(s, \tau) \in$ A denote the random target and clutter fields, respectively, such that $\mathcal{F}(T)$ and $\mathcal{F}(C)$ are in $\mathcal{L}_{\mathcal{R}}\left(L^{2}(\mathbb{R}, d t)\right)$, where $\mathcal{F}$ is the Fourier operator of the affine group as defined in (8) and (14). Define a random operator $\mathcal{E}$ as follows:

$$
\mathcal{E}=\mathcal{F}(T+C) \mathcal{W}-\mathcal{F}(T)
$$

where $\mathcal{W} \in \mathcal{L}\left(L^{2}(\mathbb{R}, d t)\right)$. Then, the unique operator $\mathcal{W}^{\text {opt }}$ from the orthogonal complement of the null space of $\mathbb{E}\left[\mathcal{F}(T+C)^{\dagger} \mathcal{F}(T+C)\right]$ to $L^{2}(\mathbb{R}, d t)$, minimizing the norm, trace $\left(\mathbb{E}\left[\mathcal{E}^{\dagger} \mathcal{E}\right]\right)$, of $\mathcal{E}$ is given by:

$$
\mathcal{W}^{\mathrm{opt}}=\left(\mathbb{E}\left[\mathcal{F}(T+C)^{\dagger} \mathcal{F}(T+C)\right]\right)^{-1} \mathbb{E}\left[\mathcal{F}(T)^{\dagger} \mathcal{F}(T)\right]
$$

where

$$
\mathbb{E}\left[\mathcal{F}(\cdot)^{\dagger} \mathcal{F}(\cdot)\right]=\mathbb{E}\left[\mathcal{F}_{+}(\cdot)^{\dagger} \mathcal{F}_{+}(\cdot)\right] \oplus \mathbb{E}\left[\mathcal{F}_{-}(\cdot)^{\dagger} \mathcal{F}_{-}(\cdot)\right] .
$$

\section{Proof: See Appendix III.}

Note that for any $X(s, \tau),(s, \tau) \in \mathrm{A}$, for which $\mathcal{F}(X)$ is a bounded random operator, $\mathbb{E}\left[\left(\mathcal{F}(X)^{\dagger} \mathcal{F}(X)\right)\right]$ is by definition a self-adjoint operator in $\mathcal{L}\left(L^{2}(\mathbb{R}, d t)\right)$. For a given orthonormal basis $\left\{\varphi_{ \pm}^{n}\right\}$ for $H_{ \pm}$, its matrix elements are given by

$$
\begin{aligned}
& \left(\mathbb{E}\left[\left(\mathcal{F}_{ \pm}(X)^{\dagger} \mathcal{F}_{ \pm}(X)\right)\right]\right)_{n m} \\
& \quad=\iiint d g d g^{\prime} C_{X}\left(g, g^{\prime}\right) \Pi_{ \pm}\left(g^{\prime-1} g\right) \varphi_{ \pm}^{n}(t) \overline{\varphi_{ \pm}^{m}}(t) d t
\end{aligned}
$$

where

$$
\mathbb{E}\left[X(g) \overline{X\left(g^{\prime}\right)}\right]=C_{X}\left(g, g^{\prime}\right), \quad g, g^{\prime} \in \text { A. }
$$


We define

$$
\mathcal{W}_{ \pm}^{\text {opt }}=\left(\mathbb{E}\left[\mathcal{F}_{ \pm}(T+C)^{\dagger} \mathcal{F}_{ \pm}(T+C)\right]\right)^{-1} \mathbb{E}\left[\mathcal{F}_{ \pm}(T)^{\dagger} \mathcal{F}_{ \pm}(T)\right]
$$

Clearly

$$
\mathcal{W}^{\mathrm{opt}}=\mathcal{W}_{+}^{\mathrm{opt}} \oplus \mathcal{W}_{-}^{\mathrm{opt}}
$$

The second-order statistics of the error

$$
\mathcal{E}=\mathcal{F}(T+C) \mathcal{W}^{\text {opt }}-\mathcal{F}(T)
$$

resulting from the minimum mean-square error filtering can be calculated from the second-order statistics of $T$ and $C$

$$
\begin{aligned}
& \mathbb{E}\left[\mathcal{E}^{\dagger} \mathcal{E}\right]=\mathbb{E}\left[\left(\mathcal{F}(T+C) \mathcal{W}^{\mathrm{opt}}\right.\right. \\
&\left.-\mathcal{F}(T))^{\dagger}\left(\mathcal{F}(T+C) \mathcal{W}^{\mathrm{opt}}-\mathcal{F}(T)\right)\right] .
\end{aligned}
$$

\section{B. Left-Affine Stationary Target and Clutter Fields}

In this subsection, we shall show that when the target and clutter fields exhibit invariance with respect to the left translations of the affine group, the preconditioning operator (37) becomes a convolution filter over the affine group. We shall call such random processes, left-affine stationary. Note that although, target and clutter fields may not be strictly left-affine stationary, under this assumption the implementation of the preconditioning operator can be simplified in return to a suboptimal solution.

Formally, a left-affine stationary process $X(g), g \in$ A can be defined as follows [31], [32]:

$$
\begin{aligned}
\mathbb{E}[X(s, \tau)] & =\text { constant, } \quad(s, \tau) \in \mathbb{A} \\
\mathbb{E}\left[X\left(g_{1}\right) \overline{X\left(g_{2}\right)}\right] & =\mathbb{E}\left[X\left(h g_{1}\right) \overline{X\left(h g_{2}\right)}\right] \quad h, g_{1}, g_{2} \in \mathcal{A} .
\end{aligned}
$$

Equation (45) implies that for all $g_{1}, g_{2} \in$ A

$$
\begin{aligned}
\mathbb{E}\left[X\left(g_{1}\right) \overline{X\left(g_{2}\right)}\right] & =C_{X}\left(g_{1}, g_{2}\right)=C_{X}\left(g_{2}{ }^{-1} g_{1}, e\right) \\
& =R_{X}\left(g_{2}{ }^{-1} g_{1}\right)=R_{X}\left(\frac{s_{1}}{s_{2}}, \frac{\tau_{1}-\tau_{2}}{s_{2}}\right)
\end{aligned}
$$

where $g_{1}=\left(s_{1}, \tau_{1}\right), g_{2}=\left(s_{2}, \tau_{2}\right)$. We shall refer to $R_{X}(s, \tau),(s, \tau) \in \mathbb{A}$ as the autocorrelation function of the process $X(s, \tau),(s, \tau) \in$ A. Note that for fixed Doppler scale $s_{1}=s_{2}=s$, the autocorrelation function becomes $R_{X}\left(1, \frac{\tau_{1}-\tau_{2}}{s}\right)$; and for fixed range $\tau_{1}=\tau_{2}, X(s, \tau),(s, \tau) \in \mathrm{A}$ is scale stationary with autocorrelation function $R_{X}\left(\frac{s_{1}}{s_{2}}, 0\right)$. Scale stationary processes are suitable to model long-term correlated, self-similar processes [22], [33].

The central fact in the study of stationary processes over groups is the existence of the spectral decomposition theorem [31]. The following theorem states the spectral decomposition of the left-affine stationary processes [31].

Theorem 5.2: A function $R_{X}(s, \tau),(s, \tau) \in \mathbb{A}$ is the autocorrelation function of an affine stationary process $X(s, \tau)$, $(s, \tau) \in \mathbb{A}$ if and only if it can be represented in the following form:

$$
R_{X}(s, \tau)=\sum_{ \pm} \operatorname{trace}\left(\Pi_{ \pm}^{\dagger}(s, \tau) \mathcal{S}_{ \pm}^{X}\right)
$$

where $\mathcal{S}_{ \pm}^{X}$ are bounded, self-adjoint and non-negative definite operators in $H_{ \pm}$.

We shall refer to $\mathcal{S}_{ \pm}^{X}$ as the spectral density operators of the process $X(s, \tau),(s, \tau) \in$ A. Clearly, if $\mathcal{F}_{ \pm}\left(R_{X}\right)$ exist, then $\mathcal{S}_{ \pm}^{X}=\mathcal{F}_{ \pm}\left(R_{X}\right) \Xi_{ \pm}$.

Let $R_{T}$ and $R_{C}$ denote the autocorrelation functions of the target reflectivity field $T$ and clutter reflectivity field $C$, respectively. Assume that both $\mathcal{F}_{ \pm}\left(R_{T}\right)$ and $\mathcal{F}_{ \pm}\left(R_{C}\right)$ exist and let

$$
\mathcal{S}_{ \pm}^{T}=\mathcal{F}_{ \pm}\left(R_{T}\right) \Xi_{ \pm} \quad \text { and } \quad \mathcal{S}_{ \pm}^{C}=\mathcal{F}_{ \pm}\left(R_{C}\right) \Xi_{ \pm}
$$

denote the spectral density operators of the target and clutter reflectivity fields, respectively.

The following theorem shows that if the target and clutter are left-affine stationary, the preconditioning operator becomes a convolution filter over the affine group; and it can be expressed in terms of the spectral density operators of the target and clutter fields in the Fourier domain of the affine group.

Theorem 5.3: Let $T(s, \tau), C(s, \tau),(s, \tau) \in$ A be zero-mean, uncorrelated, left-affine stationary processes. Let

$$
D(s, \tau)=T(s, \tau)+C(s, \tau), \quad(s, \tau) \in \text { A } .
$$

Then, the optimal linear filter $W_{\text {opt }}$, minimizing the mean-square error

$$
\int_{\text {А }} \mathbb{E}\left[|T(s, \tau)-\hat{T}(s, \tau)|^{2}\right] s^{-2} d s d \tau
$$

is given by

$$
\mathcal{W}_{ \pm}^{\text {opt }}=\mathcal{F}_{ \pm}\left(W_{\text {opt }}\right)=\Xi_{ \pm}\left[\mathcal{S}_{ \pm}^{T}+\mathcal{S}_{ \pm}^{C}\right]^{-1} \mathcal{S}_{ \pm}^{T} \Xi_{ \pm}^{-1} .
$$

The minimum mean-square error estimate $\hat{T}(s, \tau)$ of $T(s, \tau)$, $(s, \tau) \in \mathbb{A}$ is

$$
\begin{aligned}
\hat{T}(s, \tau) & =\left(D *_{\mathbf{A}} W_{\mathrm{opt}}\right)(s, \tau) \\
& =\int_{-\infty}^{\infty} \int_{0}^{\infty} D\left(s^{\prime}, \tau^{\prime}\right) W_{\mathrm{opt}}\left(\frac{s}{s^{\prime}}, \frac{\tau-\tau^{\prime}}{s^{\prime}}\right) s^{\prime-2} d s^{\prime} d \tau^{\prime} .
\end{aligned}
$$

The spectral density of the error between the signal and its estimate $E(s, \tau)=X(s, \tau)-\hat{X}(s, \tau)$ is given by

$$
\begin{aligned}
\mathcal{S}_{ \pm}^{E} & =\mathcal{S}_{ \pm}^{T}\left(I-\Xi_{ \pm}^{-1} \mathcal{F}_{ \pm}\left(W_{\mathrm{opt}}\right) \Xi_{ \pm}\right) \\
& =\mathcal{S}_{ \pm}^{T}-\mathcal{S}_{ \pm}^{T}\left(\mathcal{S}_{ \pm}^{T}+\mathcal{S}_{ \pm}^{C}\right)^{-1} \mathcal{S}_{ \pm}^{T} .
\end{aligned}
$$

Proof: The proof can be found in [34].

Note that in [34] and [35], we defined the spectral density operator as the Fourier transform of the autocorrelation function without composing it with the discrepancy operators $\Xi_{ \pm}$, 


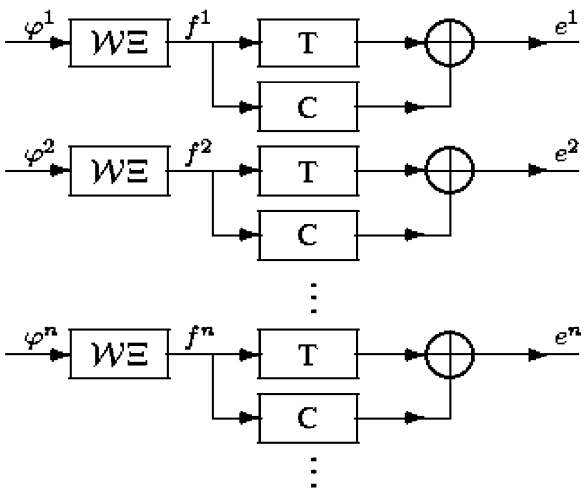

Fig. 2. Transmit and receive processing of Algorithm 1.

which leads to a representation of the Wiener filter without the discrepancy operators. Nevertheless, the representations in (53) and the ones given in [34] are equivalent.

\section{Estimation OF RANGe-DopPleR TARgET ReFLeCtivity AND DESIGN OF ClutTER REJECTING WAVEFORMS}

In this section, we shall first consider the case where measurements are contaminated by clutter only and develop methods of range-Doppler imaging and clutter rejection by transmit and receive processing. Next, we shall extend our results to the case where measurements are contaminated by both clutter and additive white noise.

\section{A. Estimation of the Target Reflectivity Function and Waveform Design in the Presence of Clutter Only}

1) Clutter Rejection in Transmit: Let the received echo signal be

$$
e(t)=\mathcal{F}(T+C) f(t) .
$$

Assume that $T$ and $C$ satisfy the conditions in Theorem 5.1 and let

$$
D(s, \tau)=T(s, \tau)+C(s, \tau), \quad(s, \tau) \in \text { A. }
$$

Applying Theorem 5.1 to (55), the minimum mean-square error estimate $\hat{T}$ of $T$ is given by

$$
\mathcal{F}_{ \pm}(\hat{T})=\mathcal{F}_{ \pm}(D) \mathcal{W}_{ \pm}^{\text {opt }} .
$$

If the target and clutter fields are left-affine stationary, then by Theorem 5.2

$$
\mathcal{W}_{ \pm}^{\mathrm{opt}}=\mathcal{F}_{ \pm}\left(W^{\mathrm{opt}}\right)
$$

Equivalently

$$
\hat{T}(s, \tau)=\left(D *_{\mathrm{A}} W^{\mathrm{opt}}\right)(s, \tau), \quad(s, \tau) \in \text { A. }
$$

The Fourier transform of the Wiener filter $W^{\mathrm{opt}}$ in (58) is given by

$$
\mathcal{F}_{ \pm}\left(W^{\mathrm{opt}}\right)=\Xi_{ \pm}\left(\mathcal{S}_{ \pm}^{T}+\mathcal{S}_{ \pm}^{C}\right)^{-1} \mathcal{S}_{ \pm}^{T} \Xi_{ \pm}^{-1}
$$

where $\mathcal{S}_{ \pm}^{T}$ and $\mathcal{S}_{ \pm}^{C}$ are the spectral density operators of the target and clutter fields, respectively. The error, $E(s, \tau)=T(s, \tau)-$ $\hat{T}(s, \tau)$, resulting from (58) is given by (53).

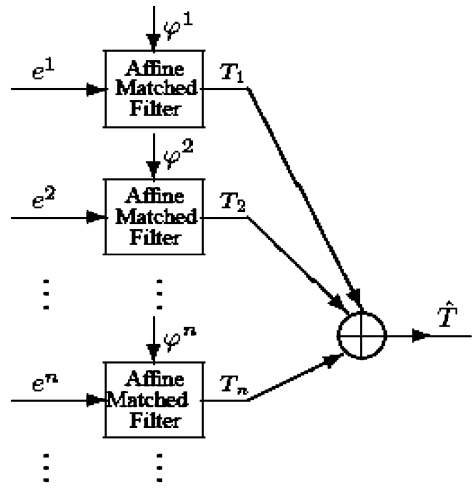

It follows from the inverse affine Fourier transform (15) and (55), (56), (59) that

$$
\begin{aligned}
\hat{T}(s, \tau) & =\sum_{n}\left\langle\mathcal{F}(\hat{T}) \Xi \varphi^{n}, \Pi(s, \tau) \varphi^{n}\right\rangle \\
& =\sum_{n}\left\langle\mathcal{F}(T+C) \mathcal{W}^{\mathrm{opt}} \Xi \varphi^{n}, \Pi(s, \tau) \varphi^{n}\right\rangle,
\end{aligned}
$$

where $\left\{\varphi^{n}\right\}$ are differentiable orthonormal basis for $L^{2}(\mathbb{R}, d t)$. Clearly, if $f^{n}=\mathcal{W}^{\mathrm{opt}} \Xi \varphi^{n}$ are the transmitted waveform, then

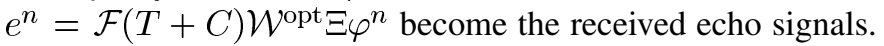
This leads to the following target reflectivity density estimation algorithm.

\section{Algorithm 1 Clutter rejection in transmit}

1: Choose a set of differentiable, orthonormal basis $\left\{\varphi_{ \pm}^{n}\right\}$ for $H_{ \pm}$, respectively.

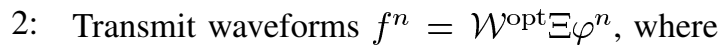
$\varphi^{n}=\varphi_{-}^{n}+\varphi_{+}^{n}, n \in \mathbb{N}$.

3: At the $n$th channel, match the received echo $e^{n}$ to $\varphi^{n}$ as follows:

$$
T_{n}(s, \tau)=\left\langle e^{n}, \Pi(s, \tau) \varphi^{n}\right\rangle .
$$

4: Coherently sum all matched filter outputs to form the image, i.e., $\hat{T}(s, \tau)=\sum_{n} T_{n}(s, \tau)$

Fig. 2 illustrates the transmit and the receiver structure and processing of Algorithm 1.

Note that in Algorithm 1, the transmitted waveforms are designed to minimize the clutter reflectivity in the reconstructed image. While there are no conditions on the orthonormal basis functions $\left\{\varphi^{n}\right\}$, apart from differentiability, clearly $\left\{\varphi^{n}\right\}$ must be in the orthogonal complement of the null space of the operator $\mathcal{W}^{\mathrm{opt}} \Xi$.

2) Clutter Suppression in Receive: For a given set of orthonormal basis, the reconstruction formula (60) can be alternatively expressed in terms of the matrix elements of the linear operators $\Pi^{\dagger}(s, \tau) \mathcal{F}(T+C)$ and $\mathcal{W}^{\text {opt }} \Xi$. Let

$$
\mathcal{A}(s, \tau)=\Pi^{\dagger}(s, \tau) \mathcal{F}(T+C),
$$




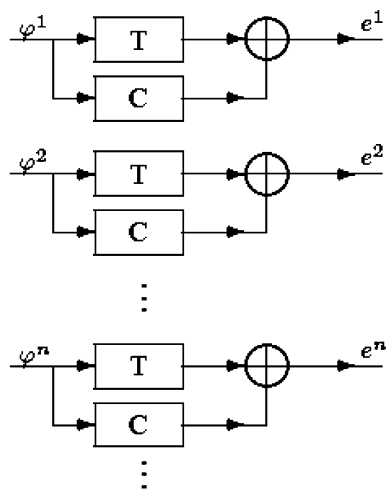

Fig. 3. Transmit and receive processing of Algorithm 2.

and

$$
\mathcal{B}=\mathcal{W}^{\text {opt } \Xi}
$$

Then

$$
\hat{T}(s, \tau)=\operatorname{trace}(\mathcal{A}(s, \tau) \mathcal{B})=\sum_{n} \sum_{p} \mathcal{A}_{n p}(s, \tau) \mathcal{B}_{p n}
$$

where

$$
\mathcal{A}_{n p}(s, \tau)=\left\langle\mathcal{F}(T+C) \varphi^{n}, \Pi(s, \tau) \varphi^{p}\right\rangle
$$

and

$$
\mathcal{B}_{p n}=\left\langle\mathcal{W}^{\text {opt }} \Xi \varphi^{p}, \varphi^{n}\right\rangle
$$

are the matrix elements of the operators $\mathcal{A}(s, \tau)$ and $\mathcal{B}$, respectively. Thus, if $\left\{\varphi^{n}\right\}$ are chosen as the transmitted waveforms, $\left\{\mathcal{F}(T+C) \varphi^{n}\right\}$ becomes the corresponding received echo signals. This observation leads to the following alternative algorithm:

\section{Algorithm 2 Clutter suppression in receive}

1: Choose a set of differentiable, orthonormal basis $\left\{\varphi_{ \pm}^{n}\right\}$ for $H_{ \pm}$.

2: $\quad$ Transmit waveforms $\varphi^{n}, n \in \mathbb{N}$, where $\varphi^{n}=\varphi_{-}^{n}+\varphi_{+}^{n}$.

3: Match the received echo $e^{n}$ at the $n$th channel to $\varphi^{p}$, $n, p \in \mathbb{N}$, i.e., $\mathcal{A}_{n p}(s, \tau)=\left\langle e^{n}, \Pi(s, \tau) \varphi^{p}\right\rangle$.

4: Compute $\mathcal{B}_{p n}$ for all $n, p \in \mathbb{N}$, the matrix elements of the operator $\mathcal{W}^{\text {opt }} \Xi$.

5: Multiply the matched filter output $\mathcal{A}_{n p}(s, \tau)$ with the weighting factor $\mathcal{B}_{p n}$.

6: Coherently sum to obtain

$$
\hat{T}(s, \tau)=\sum_{n} \sum_{p} \mathcal{A}_{n p}(s, \tau) \mathcal{B}_{p n}
$$

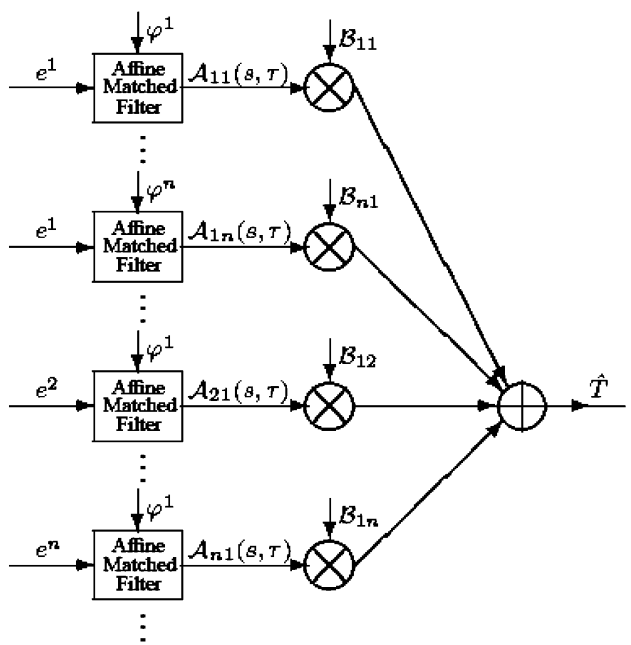

Fig. 3 illustrates the transmit and the receive structure and processing of the Algorithm 2.

\section{Remarks on Clutter Rejection/Suppression:}

1) Algorithms 1 and 2 differ mainly in the way clutter filtering is performed. Algorithm 1 filters clutter by adaptive transmit processing, i.e., by transmitting clutter rejecting waveforms, while Algorithm 2 filters clutter by adaptive receive processing, i.e., by multiplying matched filter outputs with the weighting factors $\mathcal{B}_{n m}$.

2) Algorithm 1 preconditions orthonormal basis $\left\{\varphi^{n}\right\}$ with the filter $\mathcal{W}^{\text {opt }} \Xi$ to form clutter rejecting waveforms. Algorithm 2, on the other hand, transmits $\left\{\varphi^{n}\right\}$ as waveforms.

3) For both Algorithms 1 and 2, the basis functions $\left\{\varphi^{n}\right\}$ have to span the orthogonal complement of the null space of the Wiener filter composed with the discrepancy operator, i.e., $\mathcal{W}^{\text {opt }} \Xi$. For Algorithm 1, if $\varphi^{n}$, for some $n \in \mathbb{N}$, is in the null space of $\mathcal{W}^{\mathrm{opt}} \Xi$, the echo signal resulting from the transmitted waveform $f^{n}=\mathcal{W}^{\text {opt }} \Xi \varphi^{n}$ becomes 0 . Similarly, for Algorithm 2, the weighting factor $\mathcal{B}_{n m}$, for all $m \in \mathbb{N}$, are all equal to 0 .

4) Unlike standard matched filtering, where the received echo is matched to the transmitted waveform, in both Algorithms 1 and 2, the received echo is matched to the basis functions $\left\{\varphi^{n}\right\}$. Therefore, both Algorithms 1 and 2 do not utilize the concept of auto-ambiguity, but rather the cross-ambiguity function between the transmitted waveforms $\left\{f^{n}\right\}$ and the basis functions $\left\{\varphi^{n}\right\}$.

5) Algorithm 1 is a filtered backprojection type of reconstruction formula and Algorithm 2 is a backprojection filtering type reconstruction formula. In Algorithm 1, filtering is performed in transmit and backprojection in receive, whereas in Algorithm 2 both backprojection and filtering are performed in receive.

6) While both Algorithms 1 and 2 yield the same minimum mean-square error given in (43) or (53), the computational complexity of Algorithm 1 is significantly less than that of Algorithm 2. Note that for $N$ transmitted pulses, Algorithm 1 performs $N$ matched filtering or backprojection operations, while Algorithm 2 performs $N^{2}$ backprojection 
operations. Similarly, the computation of the weighting factors $\mathcal{B}_{n m}$ require $N^{2}$ times more integration than preconditioning the waveforms in Algorithm 1.

7) Note that the orthonormal basis functions $\left\{\varphi^{n}\right\}$ do not need to be wideband signals. Therefore, the reconstruction formula (60) is applicable to a scenario where there are multiple colocated antennas operating independently, each with a limited low-resolution aperture, i.e., narrowband transmission. Nevertheless, the reconstruction formulas described in Algorithms 1 and 2 provide effective fusion of information from multiple antennas to obtain a synthetic high-resolution range-Doppler image.

\section{B. Estimation of the Range-Doppler Reflectivity Density Function in the Presence of Noise and Clutter}

It is not meaningful to design waveforms to reject/suppress additive thermal noise. We will therefore extend Algorithm 1 and address the suppression of additive noise in receive. Assume that multiple waveforms are transmitted and multiple echo signals are received as shown in Fig. 2.

$$
y^{n}(t)=\mathcal{F}(T+C) f^{n}(t)+\xi^{n}(t), \quad n \in \mathbb{N}
$$

where $f^{n}(t)$ is the $n$th transmitted waveform and $\xi^{n}(t)$ is the zero mean white noise process with variance $\sigma_{n}^{2}$ for the $n$th channel.

Assume that $\xi^{n}(t), n \in \mathbb{N}$ are uncorrelated for $n \neq m$ and that $\sum_{n} \sigma_{n}^{2}<\infty$. Let $\left\{\varphi_{ \pm}^{n}\right\}$ be differentiable, orthonormal basis for $H_{ \pm}$and $\varphi^{n}=\varphi_{+}^{n}+\varphi_{-}^{n}, n \in \mathbb{N}$; and let $f^{n}=$ $\mathcal{W}^{\text {opt }} \Xi \varphi^{n}$ be the transmitted waveforms. Using Algorithm 1, we obtain the following image:

$$
\tilde{T}(s, \tau)=\hat{T}(s, \tau)+N(s, \tau)
$$

where

$$
N(s, \tau)=\sum_{n}\left\langle\xi^{n}, \Pi(s, \tau) \varphi^{n}\right\rangle
$$

is the residual error induced by the additive white noise and $\hat{T}$ is the minimum mean-square error estimate of $T$. We shall show in Appendix IV that $N(s, \tau)$ is a left-affine stationary process with spectral density

$$
\left\langle\mathcal{S}_{ \pm}^{N} \varphi_{ \pm}^{m}, \varphi_{ \pm}^{n}\right\rangle= \begin{cases}\sigma_{n}^{2}, & \text { if } m=n \\ 0, & \text { otherwise. }\end{cases}
$$

It is straightforward to show that if the target and clutter fields are left-affine stationary, the minimum mean-square error estimate $\hat{T}$ is also left-affine stationary. Thus, one can use the second-order statistics of the error resulting from clutter rejection and Theorem 5.2, to design a minimum mean-square error convolution filter to clean residual error, $N(s, \tau),(s, \tau) \in \mathbb{A}$, in the reconstructed image. However, if the target and clutter fields are not left-affine stationary, the resulting estimate $\hat{T}$ is not necessarily left-affine stationary. As a results, the minimum meansquare error filter designed to clean the residual error $N(s, \tau)$, $(s, \tau) \in \mathbb{A}$ is not necessarily a convolution filter. To simplify our argument, we assume that the target and clutter fields are left-affine stationary.

The estimate (67) resulting from Algorithm 1 can be expressed as

$$
\tilde{T}(s, \tau)=T(s, \tau)-E(s, \tau)+N(s, \tau)
$$

where $E(s, \tau)$ is the mean-square error with spectral density

$$
\mathcal{S}_{ \pm}^{E}=\left(I-\mathcal{S}_{ \pm}^{T}\left(\mathcal{S}_{ \pm}^{T}+\mathcal{S}_{ \pm}^{C}\right)^{-1}\right) \mathcal{S}_{ \pm}^{T} .
$$

Thus, using Theorem 5.2, the minimum mean-square error estimate $\hat{\hat{T}}$ of $T$ can be obtained as follows:

$$
\begin{aligned}
\hat{\hat{T}}(s, \tau) & =\left(\tilde{T} *_{\AA} W^{\mathrm{opt} 2}\right)(s, \tau) \\
& =\int_{-\infty}^{\infty} \int_{0}^{\infty} \tilde{T}\left(s^{\prime}, \tau^{\prime}\right) W^{\mathrm{opt} 2}\left(\frac{s}{s^{\prime}}, \frac{\tau-\tau^{\prime}}{s^{\prime}}\right) s^{\prime-2} d s^{\prime} d \tau^{\prime}
\end{aligned}
$$

where $W^{\text {opt2 }}$ is the Wiener filter given by

$$
\mathcal{F}_{ \pm}\left(W^{\mathrm{opt} 2}\right)=\Xi_{ \pm}\left(\mathcal{S}_{ \pm}^{T}+\mathcal{S}_{ \pm}^{E}+\mathcal{S}_{ \pm}^{N}\right)^{-1} \mathcal{S}_{ \pm}^{T} \Xi_{ \pm}^{-1}
$$

This method is summarized in the following algorithm.

\section{Algorithm 3 Clutter rejection in transmit, noise suppression in receive}

1: Choose a set of differentiable, orthonormal basis $\left\{\varphi_{ \pm}^{n}\right\}$ for $H_{ \pm}, n \in \mathbb{N}$, where $\varphi^{n}=\varphi_{-}^{n}+\varphi_{+}^{n}$.

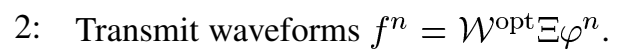

3: At the $n$th channel, match the received noisy echo $y^{n}$ to $\varphi^{n}$ as follows:

$$
T_{n}(s, \tau)=\left\langle y^{n}, \Pi(s, \tau) \varphi^{n}\right\rangle
$$

4: Coherently sum all matched filter outputs

$$
\tilde{T}(s, \tau)=\sum_{n} T_{n}(s, \tau) .
$$

5: Perform Wiener filtering over the affine group $\tilde{T} *_{\text {A }} W^{\mathrm{opt} 2}$, to suppress residual noise.

Fig. 4 illustrates the transmit and the receive structure and processing of Algorithm 3.

Note that in Algorithms 1 and 2, the filtering for clutter suppression is performed in the Fourier transform domain of the affine group. In Algorithm 3, the filtering for noise suppression is performed in the range-Doppler or affine group domain.

\section{NUMERICAL EXPERIMENTS}

We performed numerical experiments to demonstrate the validity of the proposed methods and algorithms. For ease of computation, the transmitted waveforms are derived from the Laguerre polynomials [1] and target and clutter fields are assumed to be left-affine stationary.

Let

$$
\hat{\varphi}_{+}^{n}(\omega)=L_{n-1}(\omega) e^{-\omega / 2}, \quad \omega \in \mathbb{R}^{+}, n \in \mathbb{N}
$$



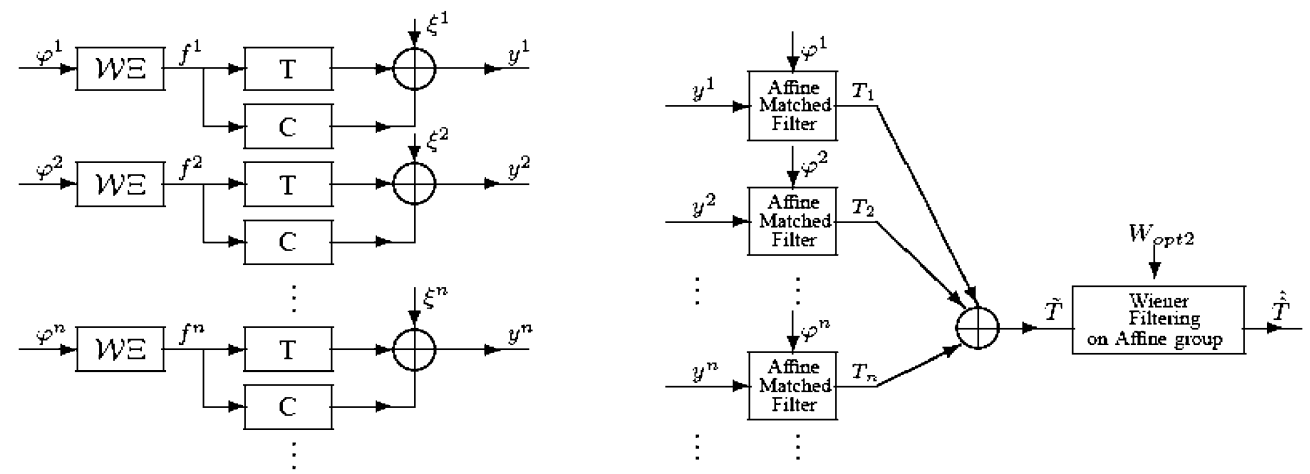

Fig. 4. Transmit and receive processing of Algorithm 3.

where $\hat{\varphi}_{+}^{n}(\omega)$ is the Fourier transform of $\varphi_{+}^{n}(t)$ and $L_{n-1}, n \in$ $\mathbb{N}$ are Laguerre polynomials defined by

$$
\begin{aligned}
L_{0}(x) & =1 \\
L_{1}(x) & =-x+1 \\
L_{n+1}(x) & =\frac{2 n+1-x}{n+1} L_{n}(x)-\frac{n}{n+1} L_{n-1}(x),
\end{aligned}
$$

It is well known that [1]

$$
\int_{0}^{\infty} e^{-x} L_{m}(x) L_{n}(x) d x= \begin{cases}1, & m=n \\ 0, & \text { else. }\end{cases}
$$

Therefore, $\left\{\hat{\varphi}_{+}^{n}\right\}$ is an orthonormal basis for $L^{2}\left(\mathbb{R}^{+}, d x\right)$. Let $\varphi_{+}^{n}$ be the standard inverse Fourier transform of $\hat{\varphi}_{+}^{n}$. Then, $\left\{\varphi_{+}^{n}\right\}$ is an orthonormal basis for $H_{+}$. Let $\hat{\varphi}_{-}^{n}(\omega)=\hat{\varphi}_{+}^{n}(-\omega)$, $\omega \in \mathbb{R}^{-}$. Then $\varphi_{-}^{n}(t)=\overline{\varphi_{+}^{n}(t)}, t \in \mathbb{R}$ and $\left\{\varphi_{-}^{n}\right\}$ is an orthonormal basis for $H_{-}$. Fig. 5 shows the first twenty basis functions, $\varphi^{n}=\varphi_{+}^{n}+\varphi_{-}^{n}$. Note that these are also the transmitted waveforms used in Algorithm 2. Fig. 6 shows the transmitted waveforms proposed in [21].

We generated realizations of the target and clutter fields based on the spectral density operators $\mathcal{S}_{ \pm}^{T}$ and $\mathcal{S}_{ \pm}^{C}$ with respect to basis $\left\{\varphi_{ \pm}^{n}\right\}$ as shown in (79) at the bottom of the page. Fig. 7 (Top) and (Middle) show realizations of the target and clutter fields with the spectral operators $\mathcal{S}^{T}$ and $\mathcal{S}^{C}$, respectively. We will use these target and clutter models to demonstrate the performance of Algorithms 1, 2, and 3.

Experiment 1: We synthesized 10 realizations of the target and clutter at various signal-to-clutter ratios (SCR) defined as $\mathrm{SCR}=20 \log _{10}\left(\sigma_{T}^{2} / \sigma_{C}^{2}\right)$, where $\sigma_{T}^{2}$ and $\sigma_{T}^{2}$ are the target and clutter variances, respectively. Each realization is generated according to spectral densities $\sigma_{T}^{2} \mathcal{S}_{ \pm}^{T}$ and $\sigma_{C}^{2} \mathcal{S}_{ \pm}^{C}$.

The Wiener filter used in Algorithms 1 and 2 is given by

$$
\Xi_{ \pm}\left(\mathcal{S}_{ \pm}^{T}+\frac{\sigma_{C}^{2}}{\sigma_{T}^{2}} \mathcal{S}_{ \pm}^{C}\right)^{-1} \mathcal{S}_{ \pm}^{T} \Xi_{ \pm}^{-1}=\Xi_{ \pm}\left(\begin{array}{cc}
I_{7 \times 7} & W_{1} \\
0_{3 \times 7} & W_{2}
\end{array}\right) \Xi_{ \pm}^{-1}
$$

where $I_{7 \times 7}$ is a 7 by 7 identity matrix, $0_{3 \times 7}$ is a $3 \times 7$ zero matrix, and $W_{1}, W_{2}$ are $7 \times 3$ and $3 \times 3$ matrices determined by $\mathcal{S}_{ \pm}^{T}, \mathcal{S}_{ \pm}^{C}$, and $\sigma_{C}^{2} / \sigma_{T}^{2}$. Fig. 8 shows the Wiener filter in the range-Doppler scale domain for $\sigma_{T}^{2} / \sigma_{C}^{2}=1$.

The transmitted waveforms in Algorithm 1 are

$$
f^{n}=\Xi\left(\mathcal{S}^{T}+\sigma_{C}^{2} / \sigma_{T}^{2} \mathcal{S}^{C}\right)^{-1} \mathcal{S}^{T} \varphi^{n} .
$$

Due to the specific structure of the matrix $\left(\mathcal{S}^{T}+\right.$ $\left.\sigma_{C}^{2} / \sigma_{T}^{2} \mathcal{S}^{C}\right)^{-1} \mathcal{S}^{T}, f^{n}=\Xi \varphi^{n}$ for $n=1, \ldots, 7 ; f^{8}=w_{8,8} \Xi \varphi^{8}$, $f^{9}=w_{9,9} \Xi \varphi^{9}, f^{10}=w_{10,10} \Xi \varphi^{10}$, and $f^{n}=0$ for $n>10$, where $\Xi \varphi^{n}, n=1,2, \ldots$ are waveforms used in [21]; and $w_{8,8}, w_{9,9}, w_{10,10}$ are the eighth, nineth, and tenth diagonal entry of the matrix $\left(\mathcal{S}^{T}+\sigma_{C}^{2} / \sigma_{T}^{2} \mathcal{S}^{C}\right)^{-1} \mathcal{S}^{T}$, respectively. Fig. 9 shows the difference between the transmitted waveforms used in Algorithm 2 and those presented in [21].

We compared Algorithms 1 and 2 with the one introduced in [21]. The results are summarized in Fig. 10. For visual comparison, we present the estimated target images in Fig. 11 for the target and clutter realizations shown in Fig. 7 (Top) and (Middle). These results show that Algorithms 1 and 2 perform better than the one introduced in [21]. In principle, Algorithms 1 and 2 should exhibit the same performance, i.e., have the same mean-square error performance. However, in Algorithm 2 the operator $\Xi=\Xi_{-}+\Xi_{+}$must be truncated to a finite sized

$$
\mathcal{S}_{ \pm}^{T}=\left(\begin{array}{ccccccccccc}
10 & 4.5 & & & & & & & & \\
4.5 & 9 & 4 & & & & & & & \\
& 4 & 8 & 3.5 & & & & & & \\
& & 3.5 & 7 & 3 & & & & & \\
& & & 3 & 6 & 2.5 & & & & \\
& & & & 2.5 & 5 & 2 & & & \\
& & & & & 2 & 4 & 1.5 & & \\
& & & & & & 1.5 & 3 & 1 & \\
& & & & & & & 1 & 2 & 0.5 \\
& & & & & & & & 0.5 & 1
\end{array}\right)
$$$$
\text { and } \quad \mathcal{S}_{ \pm}^{C}=\left(\begin{array}{ccccccc}
0_{7 \times 7} & & & & & & \\
& 4 & & & & & \\
& & 8 & & & & \\
& & & 10 & & & \\
& & & 12 & & \\
& & & & & \ddots & \\
& & & & & & 40
\end{array}\right) \text {. }
$$ 

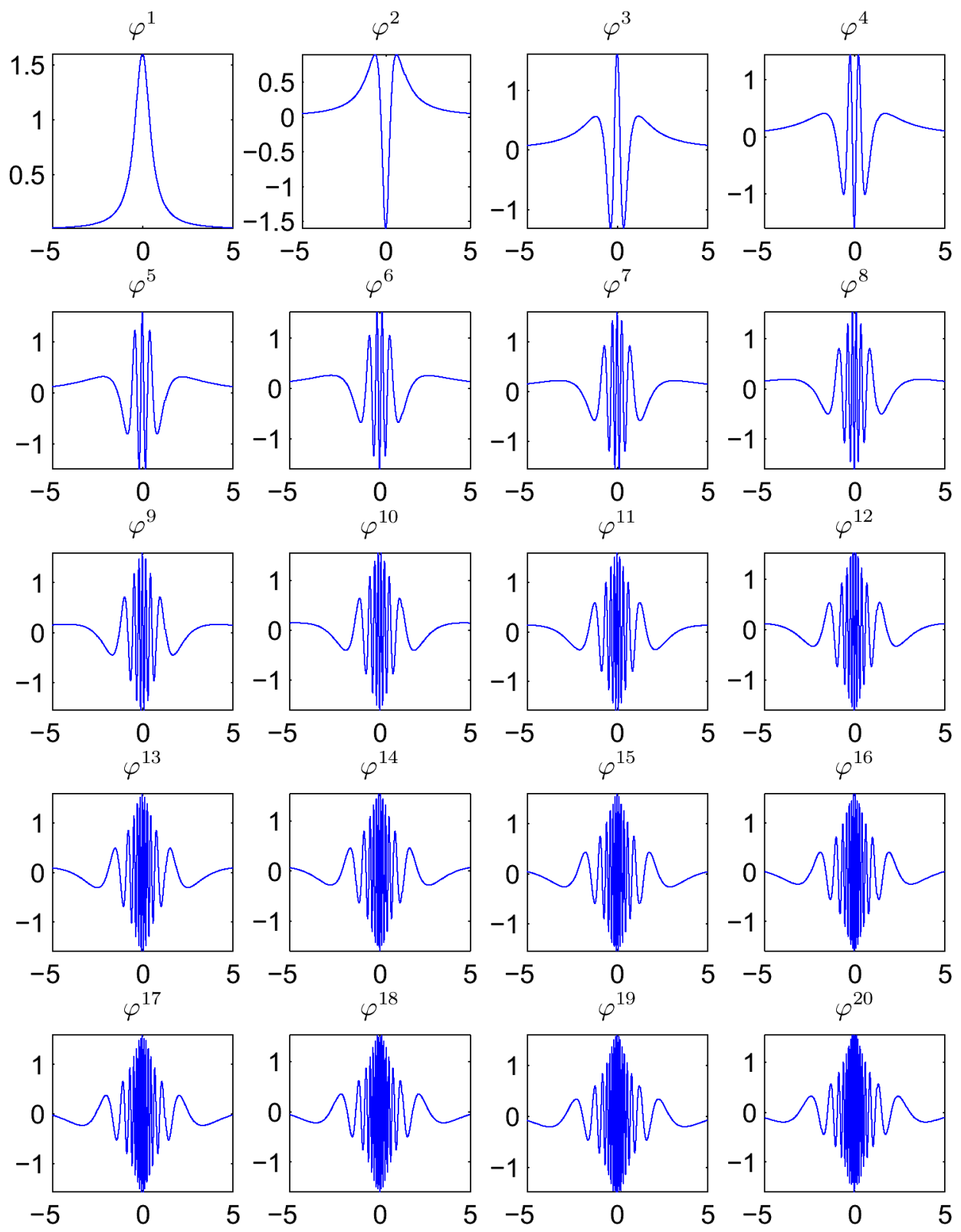

Fig. 5. Basis functions $\varphi^{n}$.

matrix for numerical implementation. This introduces a negligible degradation in the performance of Algorithm 2 as shown in Fig. 10.

Experiment 2: We performed numerical experiments to validate the performance of Algorithm 3 at various SNRs. We define the SNR as SNR $=20 \log _{10}\left(\sigma_{e_{T}}^{2} / \sigma_{n}^{2}\right)$, where $\sigma_{e_{T}}^{2}$ is the variance of the echo signal from the target and $\sigma_{n}^{2}$ is the variance of the additive noise.

Algorithm 3 involves an additional step of Wiener filtering in the range-Doppler scale or affine group domain. We observed that Algorithms 1, 2, and the one developed in [21] are all robust with respect to the additive white noise at high SNR. This can be explained by the fact that the affine matched filtering performs integration and hence averaging on the additive white noise. However, the performance of Algorithms 1, 2, and the one reported in [21] is expected to degrade for low SNR levels.
Fig. 11 shows the performance of Algorithms 1 and 3 at various SNR levels. Algorithm 3 exhibits lower performance at high SNR due to errors in numerical implementation of the Wiener filter implementation over the affine group. This numerical error is dominated by noise at low SNR levels. As shown in Fig. 11, the performance of Algorithm 3 remains unchanged while Algorithm 1 degrades as the SNR decreases.

\section{CONCLUSION}

In this paper, we introduced a group-theoretic approach to wideband range-Doppler extended target reflectivity estimation and design of clutter rejecting waveforms. This approach leads to a framework that can achieve multiple objectives simultaneously, including joint design of receive and transmit processing, rejection of clutter, suppression of noise, reduction of comptutational complexity, and use of a priori information. 

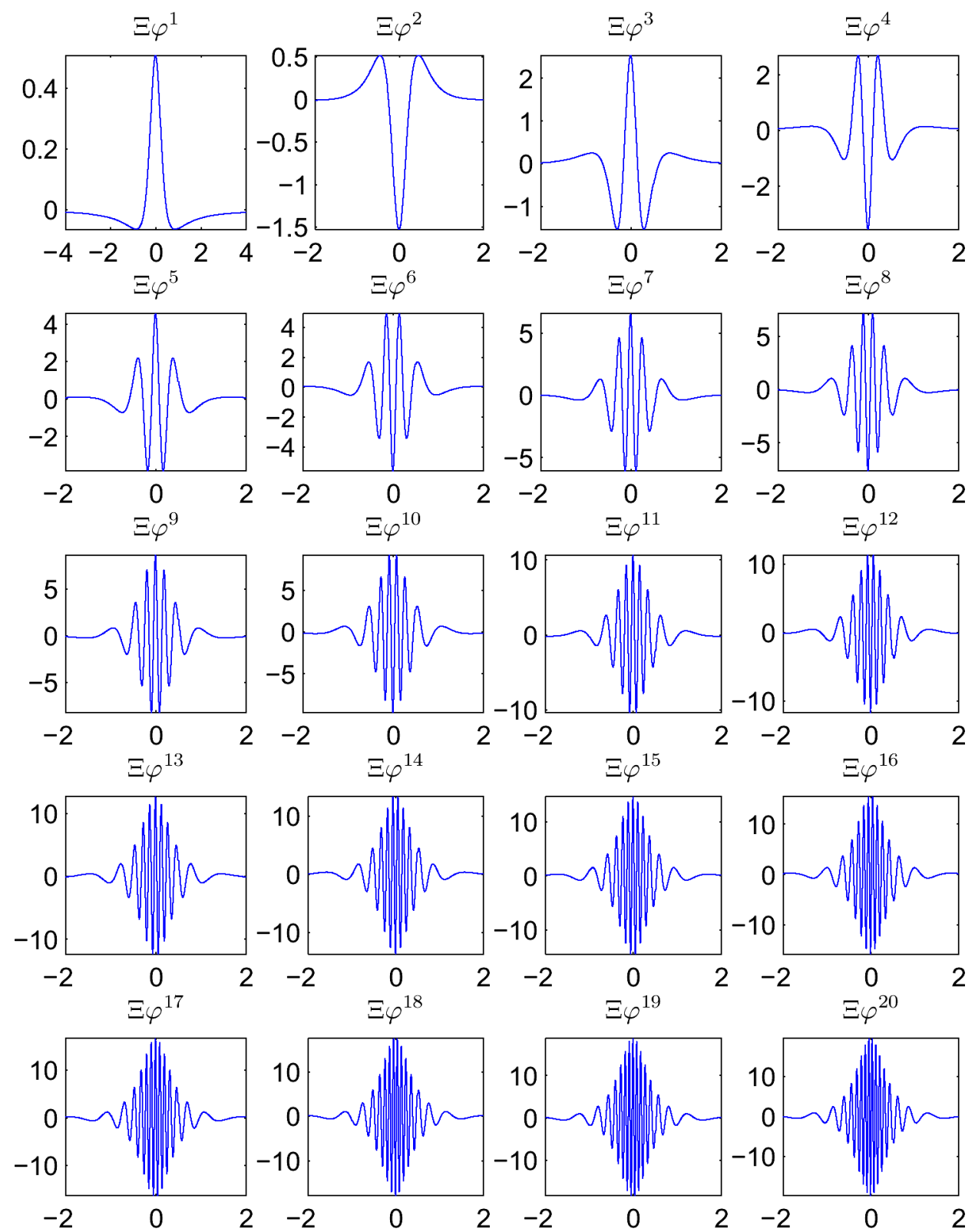

Fig. 6. Transmitted pulses used in the algorithm in [21].

We treated the affine group Fourier transform of the wideband range-Doppler extended target as an operator acting on the transmitted waveform. This leads to an exact imaging method based on the inverse Fourier transform of the affine group. Unlike the standard matched filtering approach, which is based on the backprojection of the forward map, the affine Fourier transform based approach is a filtered backprojection type reconstruction. The exactness of the reconstruction is due to the filtering operation (due the discrepancy operator) performed prior to backprojection. We designed a novel Wiener filtering method in the affine group Fourier domain and combined that with the discrepancy operator, which leads to an operator to precondition the transmit waveforms to reject clutter. We took advantage of the structure of the filtered backprojection type reconstruction and distributed processing between transmit and receive, i.e., filtering performed in transmit and backprojection performed in receive. This scheme leads to Algorithm 1, which transmits preconditioned basis functions and matches them to the basis functions. Alternatively, we re-expressed Algorithm 1 as a backprojection filtering operator to obtain Algorithm 2, which transmits orthonormal waveforms, matches them to the same orthonormal basis, and filters out clutter upon reception. We showed that Algorithm 1 provides reduced computational complexity and better utilization of the transmit power as compared to Algorithm 2. Additionally, we extended Algorithm 1 to suppress additive white noise in receive. This algorithm can be further extended to accommodate a class of strongly correlated additive noise models. The methods and algorithms introduced can be viewed as a data fusion method to form synthetic wideband images using multiple closely spaced narrowband antennas. The methodology presented in this paper can be extended to multiple distributed apertures operating in multipath environment. This study is on-going and will be reported in the near future. 

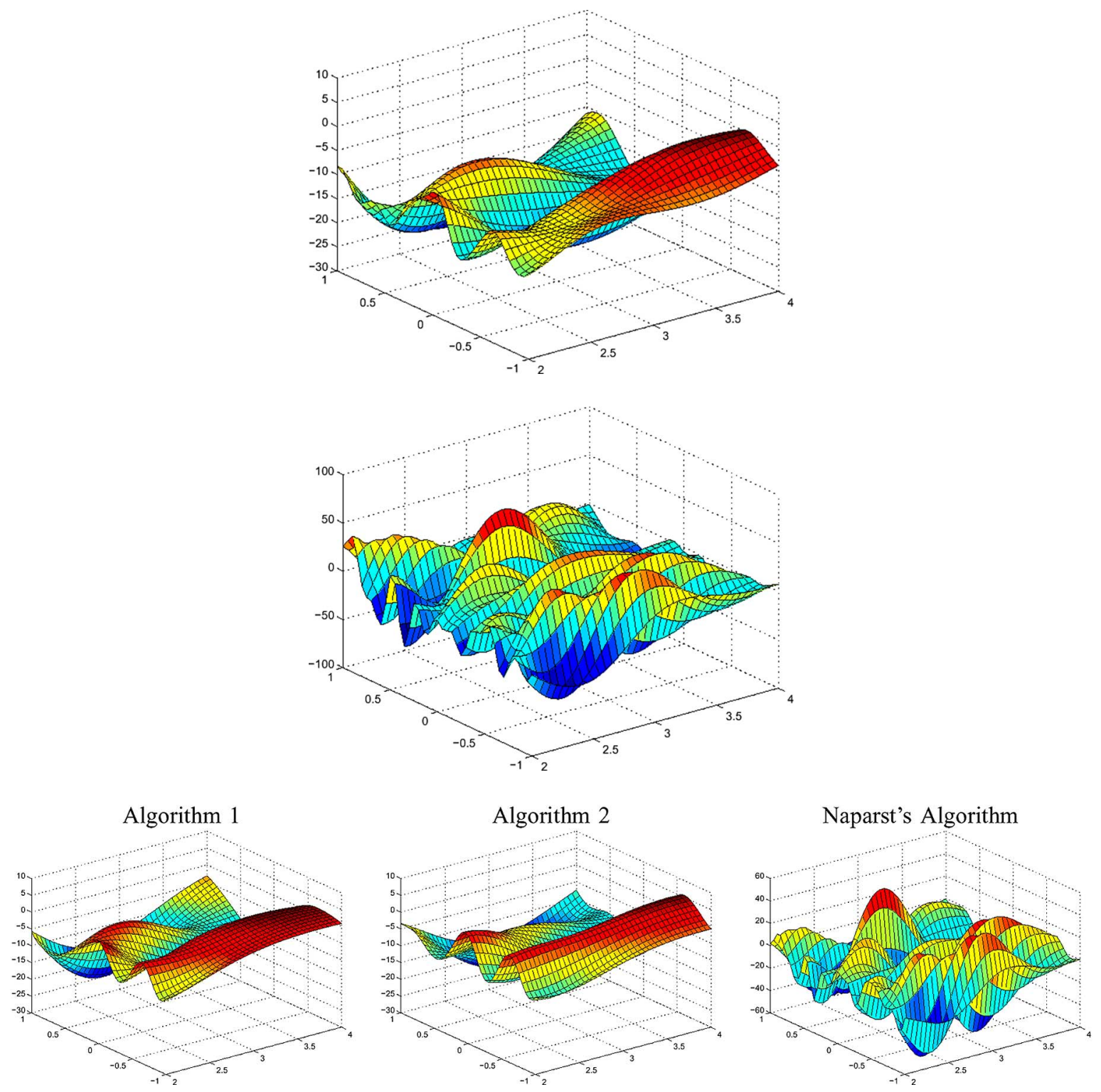

Fig. 7. Top: Target field. Middle: Clutter field. Bottom: Estimated range-Doppler target field.

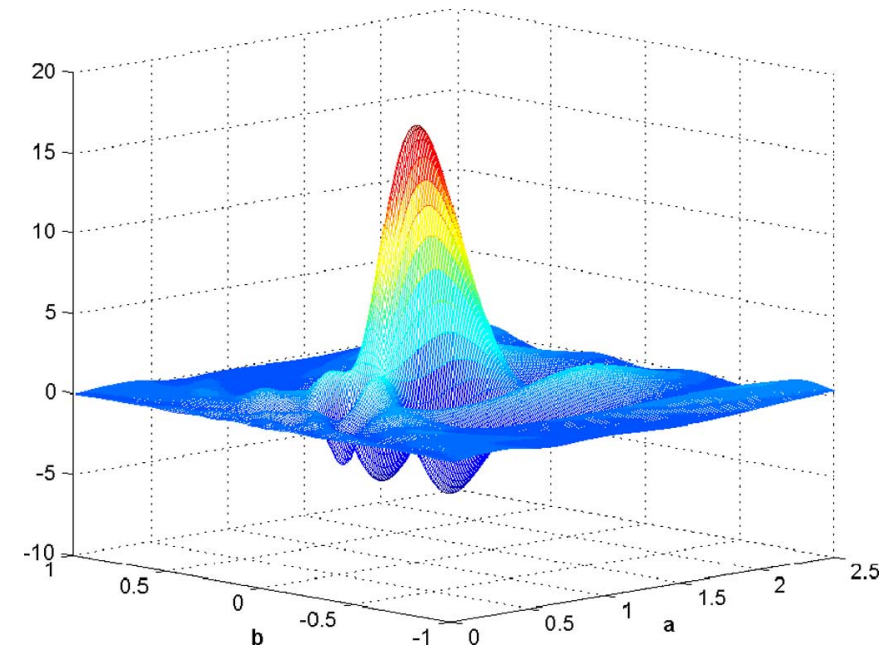

Fig. 8. Wiener filter used in Algorithms 1 and 2 shown in range-Doppler space. 

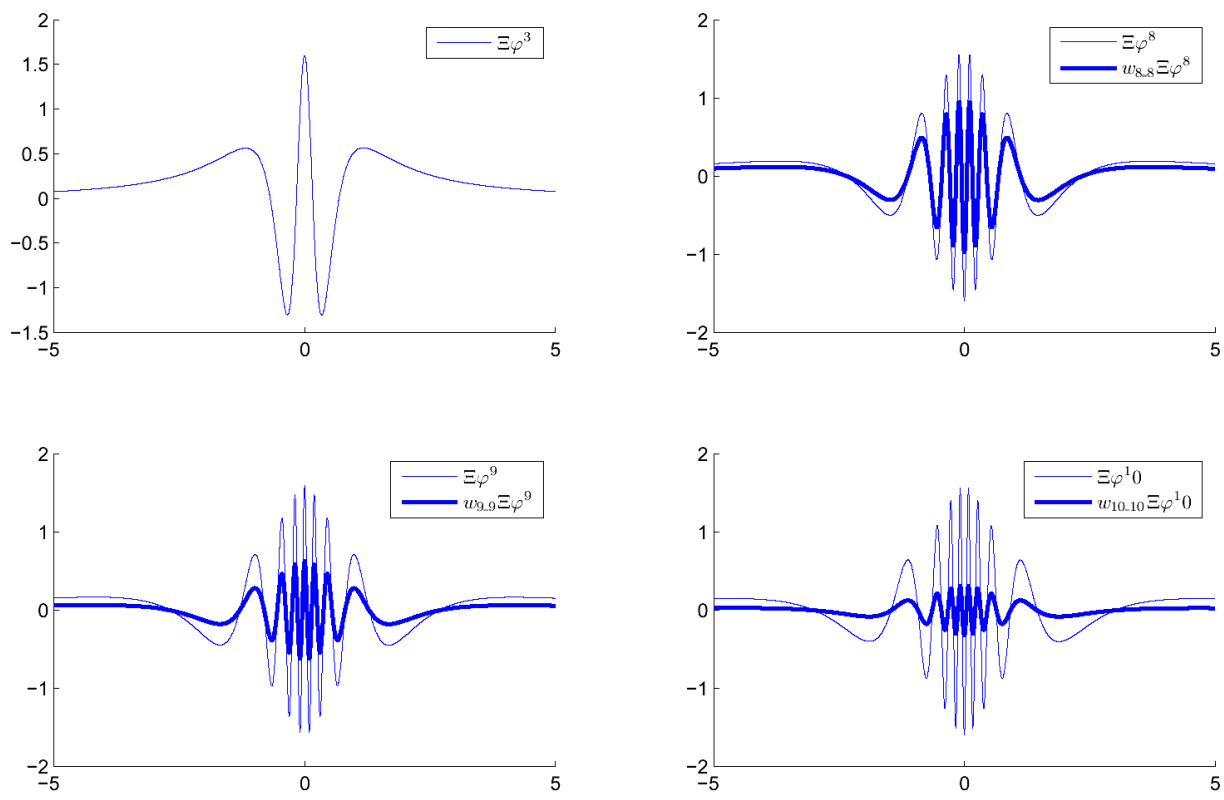

Fig. 9. Difference between the waveforms used in Algorithm 1 and the algorithm in [21] for 10-dB SCR.

\section{APPENDIX I \\ FOURIER ANALYSIS OVER GROUPS}

Fourier analysis on groups is closely related to the theory of group representations. A unitary representation of a locally compact group $G$ is a homomorphism $\Pi$ from $G$ into the group $G\left(H_{\Pi}\right)$ of unitary operators on some nonzero Hilbert space $H_{\Pi}$, i.e.,

$$
\begin{aligned}
\Pi: G & \rightarrow G\left(H_{\Pi}\right) \\
\Pi\left(g_{1} g_{2}\right) & =\Pi\left(g_{1}\right) \Pi\left(g_{2}\right), \quad g_{1}, g_{2} \in G
\end{aligned}
$$

and $\Pi(g), g \in G$ is a unitary operator on some Hilbert space $H_{\Pi}$. If $\Pi$ is invariant on a nontrivial subspace $M$ of $H_{\Pi}$, i.e., $\Pi(g) M \subset M$ for all $g \in G$, then $\Pi$ is called reducible, otherwise $\Pi$ is irreducible. For example, for each $\omega \in \mathbb{R}$

$$
\Pi_{\omega}(x)=e^{-2 \pi i \omega x}, \quad x \in \mathbb{R}
$$

acting by scalar multiplication on $H_{\Pi}=\mathbb{C}$, is an irreducible unitary representation of the additive group $(\mathbb{R},+)$.

For locally compact groups of Type I, any unitary representation can be decomposed into a direct integral of irreducible unitary representations. As a result, the space $L^{2}(G)$ is decomposed into its invariant subspaces.

The Fourier transform of a function $f \in L^{1}(G, d g)$, where $G$ is a locally compact group and $d g$ is the left-Haar measure on $G$, associated with a unitary representation $\Pi$ of $G$ is defined as a bounded operator on $H_{\Pi}$. More specifically

$$
\mathcal{F}_{\Pi}(f)=\int_{G} f(g) \Pi(g) d g
$$

where the operator-valued integral is interpreted as

$$
\left\langle\mathcal{F}_{\Pi}(f) u, v\right\rangle=\int_{G} f(g)\langle\Pi(g) u, v\rangle d g, \quad \text { for all } u, v \in H_{\Pi} \text {. }
$$

Under this definition, the Fourier transform of $f(x) \in L^{1}(\mathbb{R})$ associated with the representation defined in (83) is given by

$$
\mathcal{F}_{\Pi_{\omega}}(f)=\int_{\mathbb{R}} f(x) e^{-2 \pi i \omega x} d x, \quad \forall \omega \in \mathbb{R}
$$

which is the same as the classical Fourier transform on $\mathbb{R}$.

For a locally compact group $G$ with left-Haar measure $d g$, the convolution of $F_{1}, F_{2} \in L^{1}(G, d g)$ is defined as

$$
\left(F_{1} * F_{2}\right)(g)=\int_{G} F_{1}(h) F_{2}\left(h^{-1} g\right) d h, \quad g \in G .
$$

An important property of the Fourier transforms over groups is that the group convolution becomes operator composition in Fourier space. More specifically

$$
\mathcal{F}_{\Pi}\left(F_{1} * F_{2}\right)=\mathcal{F}_{\Pi}\left(F_{1}\right) \mathcal{F}_{\Pi}\left(F_{2}\right)
$$

For two unitary representations $\Pi_{1}, \Pi_{2}$ of $G$, if there exists a unitary operator $U: H_{\Pi_{1}} \rightarrow H_{\Pi_{2}}$ such that $\Pi_{2}(x)=$ $U \Pi_{1}(x) U^{-1}$ for all $x \in G$, then $\Pi_{1}$ and $\Pi_{2}$ are called equivalent representations. The collection of all nonequivalent irreducible representation classes of $G$ is denoted by $\hat{G}$ and is called the dual of group $G$. The collection of Fourier transforms $\left\{\mathcal{F}_{\lambda}(f)\right\}$ for all $\lambda \in \hat{G}$ is called the spectrum of the function $f$. For a separable locally compact group of Type I, the Fourier synthesis formula exists. More specifically

$$
f(g)=\int_{\hat{G}} \operatorname{trace}\left(\Pi_{\lambda}^{*}(g) \mathcal{F}_{\lambda}(f) \Xi_{\lambda}\right) d \nu(\lambda),
$$

where $d \nu(\lambda)$ is the Plancherel measure of the dual group $\hat{G}$ and $\Xi_{\lambda}$ is a self-adjoint, positive-definite operator defined on $H_{\Pi_{\lambda}}$ satisfying

$$
\Xi_{\lambda} \Pi_{\lambda}(g)=\Delta(g) \Pi_{\lambda}(g) \Xi_{\lambda}, \quad \text { for all } g \in G
$$

where $\Delta(g)$ is the modular function on $G$. 


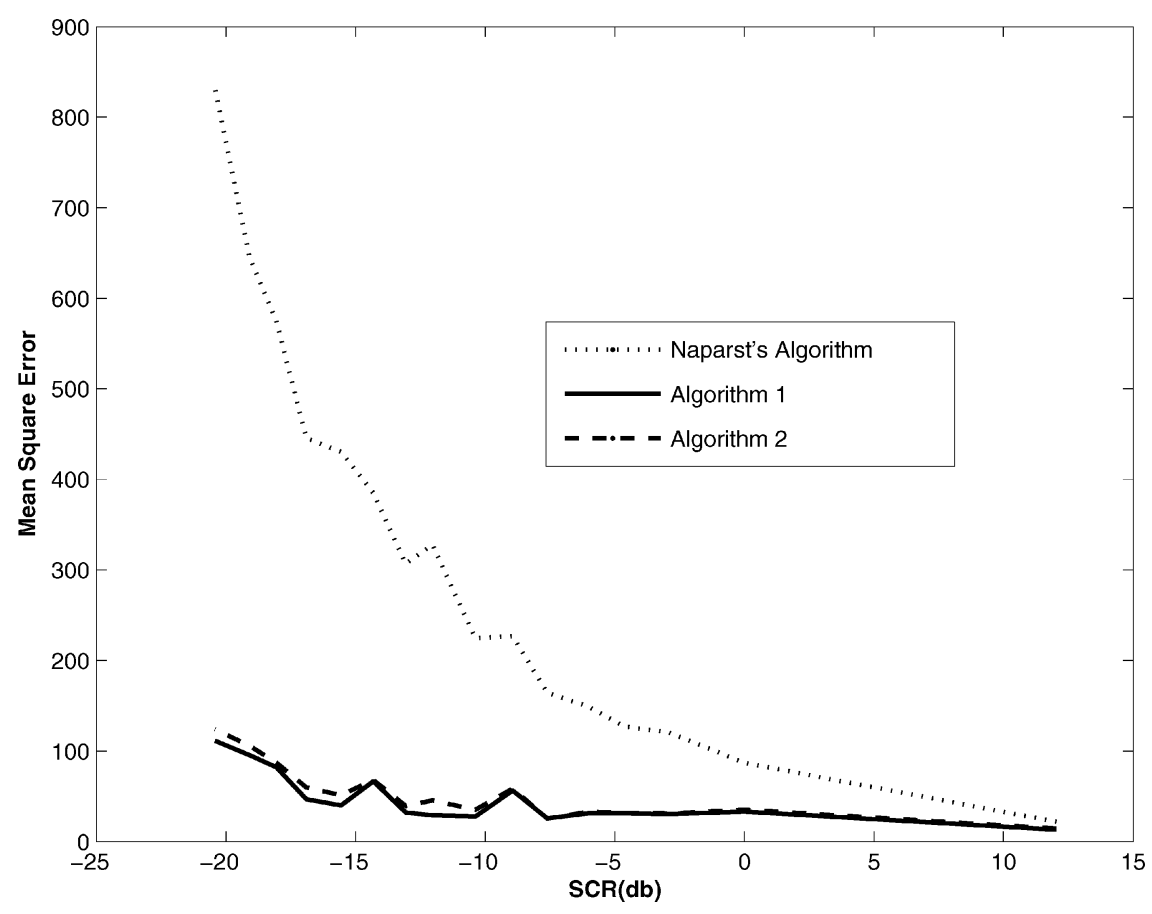

Fig. 10. Comparison of Algorithms 1, 2 and the algorithm in [21].

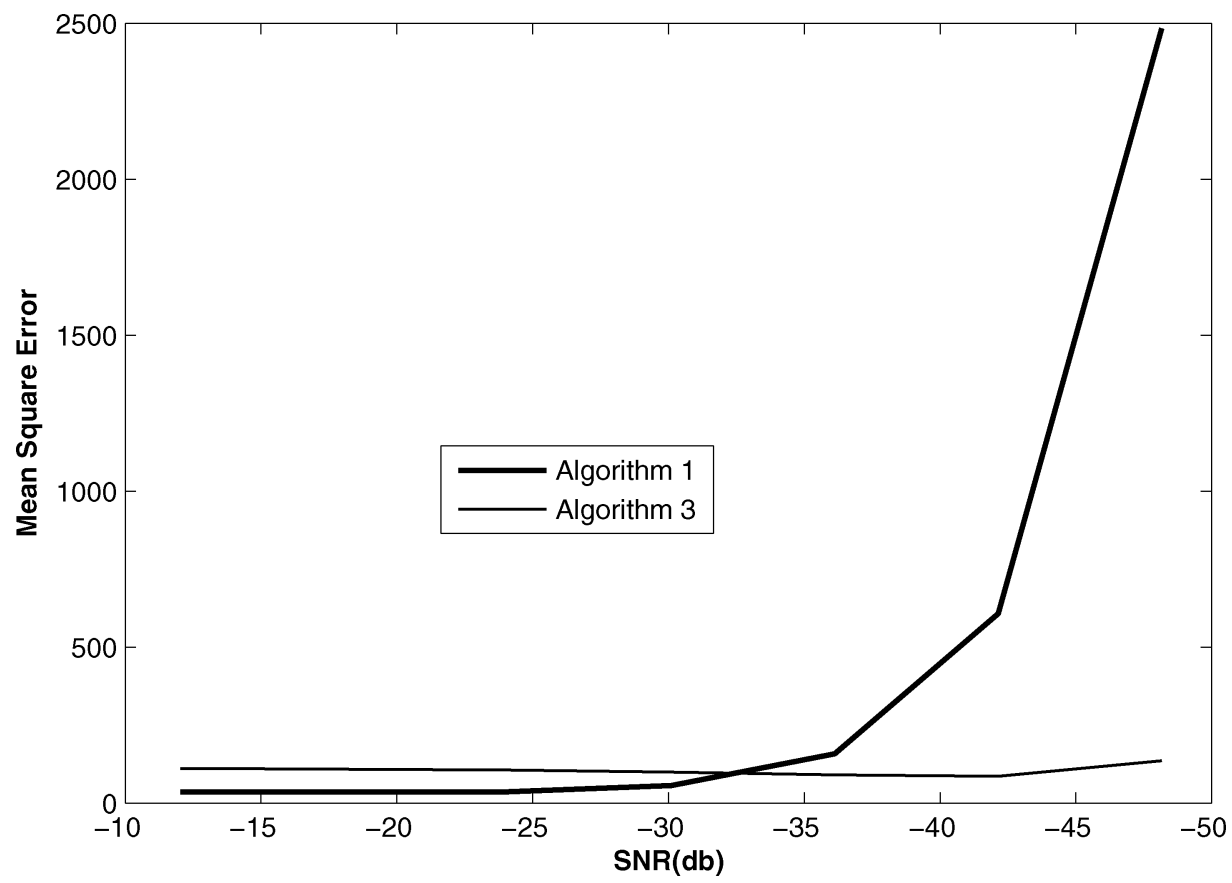

Fig. 11. Comparison of Algorithm 1 and 3 for different SNR levels.

\section{APPENDIX II}

The following lemma is used repeatedly throughout the paper. The proof is straightforward and can be found in many books. See, for example [10, Ch. 2].

Lemma: Let $\varphi^{i} \in L^{2}(\mathbb{R}, d t), i=1,2,3,4$, then the following identity holds if all the integrals exist:

$\int_{\text {丹 }}\left\langle\Pi(g) \varphi^{1}, \varphi^{2}\right\rangle\left\langle\varphi^{4}, \Pi(g) \varphi^{3}\right\rangle d g=\sum_{ \pm}\left\langle\varphi_{ \pm}^{4}, \varphi_{ \pm}^{2}\right\rangle\left\langle\Xi_{ \pm}^{-1} \varphi_{ \pm}^{1}, \varphi_{ \pm}^{3}\right\rangle$ where $\varphi_{ \pm}^{i}, i=1,2,3,4$ are the orthogonal components of $\varphi^{i}$ in $H_{ \pm}$.

\section{APPENDIX III}

Proof of Theorem 5.1: Let

$$
J(\mathcal{W})=\operatorname{trace}\left(\mathbb{E}\left[\mathcal{E}^{\dagger} \mathcal{E}\right]\right) .
$$

We will use calculus of variations to calculate the variational derivative of the functional $J$ with respect to the operator $\mathcal{W}$. 
Let $\left\{\varphi^{n}\right\}$ be an orthonormal basis for $L^{2}(\mathbb{R}, d t)$. Then, $J$ can be alternatively expressed as

$$
\begin{aligned}
& J(\mathcal{W})=\sum_{n} \mathbb{E}\left[\left\langle\mathcal{E}^{\dagger} \mathcal{E} \varphi^{n}, \quad \varphi^{n}\right\rangle\right] \\
&=\sum_{n} \mathbb{E}\left[\left\langle(\mathcal{F}(T+C) \mathcal{W}-\mathcal{F}(T)) \varphi^{n}\right.\right. \\
&\left.\left.(\mathcal{F}(T+C) \mathcal{W}-\mathcal{F}(T)) \varphi^{n}\right\rangle\right]
\end{aligned}
$$

$J$ attains its minimum at $\mathcal{W}$, if

$$
J\left(\mathcal{W}+\mathcal{W}_{\epsilon}\right)-J(\mathcal{W})=\mathcal{O}\left(\left\|\mathcal{W}_{\epsilon}^{2}\right\|\right)
$$

for all $\mathcal{W}_{\epsilon}$ and $\varphi \in L^{2}(\mathbb{R}, d t)$. Equation (94) implies that for all $\varphi^{n} \in L^{2}(\mathbb{R}, d t)$ and $\mathcal{W}_{\epsilon}$

$0=\sum_{n} 2 \operatorname{Re} \mathbb{E}\left[\left\langle\mathcal{W}_{\epsilon}^{\dagger}(\mathcal{F}(T+C))^{\dagger}(\mathcal{F}(T+C) \mathcal{W}-\mathcal{F}(T)) \varphi^{n}, \varphi^{n}\right\rangle\right]$

Equivalently

$$
\begin{aligned}
0 & =2 \operatorname{Re} \mathbb{E}\left[\left\langle\mathcal{W}_{\epsilon}^{\dagger}(\mathcal{F}(T+C))^{\dagger}(\mathcal{F}(T+C) \mathcal{W}-\mathcal{F}(T)) \varphi^{n}, \varphi^{n}\right\rangle\right] \\
& =\mathbb{E}\left[(\mathcal{F}(T+C))^{\dagger}(\mathcal{F}(T+C) \mathcal{W}-\mathcal{F}(T))\right] .
\end{aligned}
$$

Since $T$ and $C$ are statistically independent

$$
\mathcal{W}=\left(\mathbb{E}\left[(\mathcal{F}(T+C))^{\dagger} \mathcal{F}(T+C)\right]\right)^{-1} \mathbb{E}\left[(\mathcal{F}(T))^{\dagger} \mathcal{F}(T)\right] .
$$

\section{APPENDIX IV}

Let $\xi(t)$ be the zero-mean white noise process with variance $\sigma^{2}$. It is straightforward to show that for any $f \in L^{2}(\mathbb{R}, d t)$, the process $N(s, \tau)=\langle\xi, \Pi(s, \tau) f\rangle,(s, \tau) \in \mathbb{A}$ is a zero mean, stationary process over the affine group with the following autocorrelation function:

$$
R(s, \tau)=\sigma^{2}\langle f, \Pi(s, \tau) f\rangle, \quad(s, \tau) \in \text { A. }
$$

For a given orthonormal differentiable basis $\left\{\varphi_{ \pm}^{n}\right\}$ of $H_{ \pm}$, using the lemma in Appendix II, we can show that the matrix elements of the spectral density operators $\mathcal{S}_{ \pm}$of $N(s, \tau),(s, \tau) \in$ A can be written as

$$
\begin{aligned}
& \left\langle\mathcal{S}_{ \pm} \varphi_{ \pm}^{m}, \varphi_{ \pm}^{n}\right\rangle \\
& =\left\langle\mathcal{F}_{ \pm}(R) \Xi_{ \pm} \varphi_{ \pm}^{m}, \varphi_{ \pm}^{n}\right\rangle \\
& =\sigma^{2} \int_{-\infty}^{\infty} \int_{0}^{\infty}\langle f, \Pi(s, \tau) f\rangle\left\langle\Pi_{ \pm}(s, \tau) \Xi_{ \pm} \varphi_{ \pm}^{m}, \varphi_{ \pm}^{n}\right\rangle s^{-2} d s d \tau \\
& =\sigma^{2}\left\langle\varphi_{ \pm}^{m}, f_{ \pm}\right\rangle\left\langle f_{ \pm}, \varphi_{ \pm}^{n}\right\rangle
\end{aligned}
$$

where $f_{ \pm}$are the orthogonal components of $f$ in $H_{ \pm}$.

Let $N^{m}(s, \tau)=\left\langle\xi^{m}, \Pi(s, \tau) \varphi^{m}\right\rangle$. Then, $N^{m}, m \in \mathbb{N}$ are zero mean, affine stationary processes with the autocorrelation function

$$
R_{N^{m}}(s, \tau)=\sigma_{m}^{2}\left\langle\varphi^{m}, \Pi(s, \tau) \varphi^{m}\right\rangle ;
$$

and the spectral density operators

$$
\begin{aligned}
\left\langle\mathcal{S}_{ \pm}^{N^{m}} \varphi_{ \pm}^{l}, \varphi_{ \pm}^{k}\right\rangle & =\sigma_{m}^{2}\left\langle\varphi_{ \pm}^{l}, \varphi_{ \pm}^{m}\right\rangle\left\langle\varphi_{ \pm}^{m}, \varphi_{ \pm}^{k}\right\rangle \\
& = \begin{cases}\sigma_{m}^{2}, & \text { if } l=k=m \\
0, & \text { otherwise }\end{cases}
\end{aligned}
$$

Since $\xi^{m}(t), m \in \mathbb{N}$ are uncorrelated, $N^{m}, m \in \mathbb{N}$ are also uncorrelated. It follows from $\sum_{m} \sigma_{m}^{2}<\infty$ that $\sum_{m} N^{m}(s, \tau)$ converges in the mean square sense. Let

$$
N(s, \tau)=\sum_{m} N^{m}(s, \tau)=\sum_{m}\left\langle\xi^{m}(t), \Pi(s, \tau) \varphi^{m}\right\rangle .
$$

Then, $N$ is a zero mean, affine stationary process with the spectral density operators

$$
\mathcal{S}_{ \pm}^{N}=\sum_{m} \mathcal{S}_{ \pm}^{N^{m}}
$$

Hence,

$$
\left\langle\mathcal{S}_{ \pm}^{N} \varphi_{ \pm}^{m}, \varphi_{ \pm}^{n}\right\rangle= \begin{cases}\sigma_{n}^{2}, & \text { if } m=n \\ 0, & \text { otherwise }\end{cases}
$$

\section{ACKNOWLEDGMENT}

The first author would like to thank Dr. Trond Varslot and Dr. C. Evren Yarman for numerous technical discussions.

\section{REFERENCES}

[1] M. Abramowitz and I. A. Stegun, Eds., "Orthogonal polynomials," in Handbook of Mathematical Functions with Formulas, Graphs, and Mathematical Tables, 9th ed. New York: Dover, 1972, ch. 22, pp. 771-802.

[2] R. H. Barker, "Group synchronizing of binary digital systems," in Communication Theory, W. Jackson, Ed.. New York: Academic, 1953, pp. 273-287.

[3] M. R. Bell, "Information theory and radar waveform design," IEEE Trans. Inf. Theory, vol. 39, no. 5, pp. 1578-1597, Sep. 1993

[4] R. E. Blahut, W. Miller, and C. H. Wilcox, Radar and Sonar, Part I. New York: Springer-Verlag, 1991

[5] R. E. Blahut, Theory of Remote Image Formation. Cambridge, U.K.: Cambridge Univ. Press, 2004.

[6] B. Borden, Radar Imaging of Airborne Targets. New York: Institute of Physics Publishing, 1999.

[7] C. Chang and M. R. Bell, "Frequency-coded waveforms for enhanced delay-Doppler resolution," IEEE Trans. Inf. Theory, vol. 49, no. 11, pp. 2960-2971, Nov. 2003.

[8] J. P. Costas, "A study of a class of detection waveforms having nearly ideal range-Doppler ambiguity properties," Proc. IEEE, vol. 72, no. 8 , pp. 996-1009, Aug. 1984.

[9] C. E. Cook and M. Bernfeld, Radar Signals. New York: Academic, 1967.

[10] I. Daubechies, Ten Lectures on Wavelets. Philadelphia, PA: SIAM, 1992.

[11] R. L. Frank, "Polyphase codes with good nonperiodic correlation properties," IEEE Trans. Inf. Theory, vol. PGIT-9, no. 1, pp. 43-45, Jan. 1963.

[12] D. A. Garren, M. K. Osborn, A. C. Odom, J. S. Goldstein, S. U. Pillai, and J. R. Guerci, "Enhanced target detection and identification via optimized radar transmission pulse shape," Proc. Inst. Elec Eng., Radar, Sonar and Navigation, vol. 148, no. 3, pp. 130-138, Jun. 2001.

[13] J. R. Guerci and P. Grieve, "Optimum Matched Illumination-Reception Radars," U.S. 5121 125, and U.S. Patent 5175 552, Jun. Dec. 1992.

[14] D. T. Gjessing, "Adaptive techniques for radar detection and identification of objects in an ocean environment," IEEE J. Ocean. Eng., vol. 6, no. 1, pp. 5-17, Jan. 1981

[15] —, Target Adaptive Matched Illumination Radar: Principles and Applications. Stevenage, U.K.: Peter Peregrinus, 1986.

[16] S. W. Golomb and H. Taylor, "Constructions and properties of Costas arrays," Proc. IEEE, vol. 72, no. 9, pp. 1143-1163, Sep. 1984.

[17] _ , "Algebraic construction of Costas arrays," J. Comb. Theory, ser A, vol. 37, pp. 13-21, 1984.

[18] J. C. Guey and M. R. Bell, "Diversity waveform sets for delay-Doppler imaging," IEEE Trans. Inf. Theory, vol. 44, no. 4, pp. 1504-1522, Jul. 1998. 
[19] E. G. Kalnins and W. Miller, "A note on group contractions and radar ambiguity functions," in Radar and Sonar, M. Bernfeld, R. Blahut, and A. Grunbaum, Eds. New York: Springer-Verlag, 1992, IMA Volumes in Mathematics and its Applications, pt. II.

[20] W. Miller, "Topics in harmonic analysis with applications to radar and sonar," in Radar and Sonar, R. E. Blahut, W. Miller, and C. H. Wilcox, Eds. New York: Springer-Verlag, 1991, pp. 66-168.

[21] H. Naparst, "Dense target signal processing," IEEE Trans. Inf. Theory, vol. 37, no. 2 , pp. 317-327, Mar. 1991.

[22] C. J. Nuzman and H. V. Poor, "Linear estimation of self-similar processes via Lamberti's transformation,” J. Appl. Probab., vol. 32, no. 2, Jun. 2000.

[23] S. U. Pillai, H. S. Oh, D. C. Youla, and J. R. Guerci, "Optimum transmit-receiver design in the presence of signal-dependent interference and channel noise," IEEE Trans. Inf. Theory, vol. 46, no. 2, pp. 577-584, Mar. 2000.

[24] L. Rebollo-Neira, A. Pastino, and J. Fernandez-Rubio, "Reconstruction of the joint time-delay Doppler-scale reflectivity density in the wideband regime: A frame theory based approach," J. Math. Phys., vol. 41, no. 8, pp. 5325-5341, 2000.

[25] R. Sivaswamy, "Digital and analog subcomplementary sequences for pulse compression," IEEE Trans. Aerosp. Electron. Syst., vol. AES-14, pp. 343-350, 1978

[26] _ "Multiphase complementary codes," IEEE Trans. Inf. Theory, vol. IT-24, no. 5, pp. 546-552, Sep. 1978.

[27] S. M. Sowalem and A. H. Tewfik, "Waveform selection in radar target classification," IEEE Trans. Inf. Theory, vol. 46, no. 3, pp. 1014-1029, May 2000.
[28] D. A. Swick, A Review of Wideband Ambiguity Functions Naval Res. Lab., Washington, DC, 1969, NRL Rep. 6994.

[29] J. D. Taylor, Introduction to Ultra-Wideband Radar Systems. Boca Raton, FL: CRC, 1995.

[30] N. J. Vilenkin, Special Functions and the Theory of Representations. Providence, RI: Amer. Math. Soc., 1998.

[31] A. M. Yaglom, "Second order homogeneous random fields," in Fourth Berkley Symp. Mathematical Statistics and Probability, Berkeley, CA, 1961, vol. 2, pp. 593-622.

[32] B. Yazıcı and R. L. Kashyap, "Affine stationary processes with applications to fractional Brownian motion," in Proc. 1997 Int. Conf. Acoustics, Speech, and Signal Processing, Atlanta, GA, Apr. 1997, vol. 5, pp. 3669-3672.

[33] _ _Second order stationary, self-similar models for $1 / \mathrm{f}$ processes," IEEE Trans. on Signal Processing, pp. 396-410, Feb. 1997.

[34] B. Yazıc1, "Stochastic deconvolution over groups," IEEE Trans. Inf. Theory, vol. 50, no. 3, pp. 494-510, Mar. 2004.

[35] _ "Stochastic deconvolution over groups for inverse problems in imaging," in Proc. 2003 IEEE Int. Conf. Acoustics, Speech, and Signal Processing, Hong Kong, China, Apr. 2003, vol. 6, pp. 577-580.

[36] L. G. Weiss, "Wavelets and wideband correlation processing," IEEE Signal Processing Mag., vol. 11, no. 1, pp. 13-32, Jan. 1994.

[37] C. H. Wilcox, "The Synthesis Problem for Radar Ambiguity Functions," MRC Tech., Summary Rep., 1960. 